\title{
On the Use of the Analogue Transformation Acoustics in Aeroacoustics
}

\author{
Umberto Iemma and Giorgio Palma \\ Department of Engineering, Roma Tre University, Via Vito Volterra, No. 62, 00146 Rome, Italy \\ Correspondence should be addressed to Giorgio Palma; giorgio.palma@uniroma3.it
}

Received 23 June 2017; Revised 20 October 2017; Accepted 29 October 2017; Published 16 November 2017

Academic Editor: Michele Brun

Copyright (C) 2017 Umberto Iemma and Giorgio Palma. This is an open access article distributed under the Creative Commons Attribution License, which permits unrestricted use, distribution, and reproduction in any medium, provided the original work is properly cited.

\begin{abstract}
The objective of the paper is the assessment of the Analogue Transformation Acoustics (ATA) in the design of acoustic metamaterial for aeronautical applications. The work focuses on the consistency of the background flow resulting from the application of the ATA with the equations governing the potential aerodynamics. Indeed, in case of acoustic perturbations propagating within moving media, the convective terms in the governing equations are responsible for the failure of formal invariance under the action of conformal mappings. The ATA approach overcomes this limitation, introducing the possibility of handling the convective form of the wave equation in a straightforward and elegant way. It is based on the concept of analogue space-time and fully relies on the analytical tools of Lorentzian differential geometry. The present paper analyses the relationship between the analogue velocity field with a realistic potential flow. The method is validated through numerical simulations using two widely assessed acoustic cloaking problems. The preliminary results obtained show that the use of numerical, quasi-conformal mappings can lead to transformed streamlines negligibly deviating from those of the potential velocity field satisfying the fluid-dynamic conservation laws, but with incompatible intensity of the local velocity.
\end{abstract}

\section{Introduction}

Since the early achievement of electromagnetic invisibility, published in 2006 by Pendry et al. [1] and Leonhardt [2], and the subsequent porting of the concept to acoustics by Cummer and Schurig [3], the design of acoustic metamaterials based on coordinate transformations has one of the most effervescent areas of research worldwide. The fundamentals of the coordinate transformation approach are based on the observation that mass and momentum equations for an inviscid fluid at rest under small pressure perturbation exhibit a strict formal analogy with the single polarization Maxwell equations, provided that suitable variables exchange is introduced. Most of the research activity has focused on the achievement of acoustic invisibility, or cloaking, that is, the total abatement of the scattering effects induced by an obstacle impinged by an acoustic perturbation. The standard approach (a.k.a. STA, Standard Transformation Acoustics), directly imported from electromagnetism, through the exploitation of the formal invariance of governing equations under coordinates transformations, reinterprets the components of the transformation metrics as the mechanical properties of an ideal material, able to attain the target behavior. The resulting concept of acoustic metamaterial (or metafluid, Norris $[4,5]$ ) has disclosed an incredible potential of development of breakthrough technologies, enthusiastically welcomed by the research and industrial community in the quest for highly innovative, unconventional noise mitigation devices and concepts. Unfortunately, the extension of the same concepts to aeroacoustics has turned out to be not a trivial task, because of the different structure of the governing equations, characterized by the presence of the background aerodynamic convection. The latter introduces transport terms in the governing equations which yield, under suitable assumptions, the convective forms of the wave operator. The arising mixed space-time derivatives make the formal invariance of the governing equations under coordinate transformations vanish.

Acoustic metamaterials received enormous attention since their appearance in 2006 and rapidly became a very 
popular topic. A detailed bibliographic review is beyond the scope of this paper; however it is worth mentioning some attempts to address the aeroacoustic cloaking problem, that is, in the presence of flow or motion of scatterers and sources, which can be found in literature. In Huang et al. [6] a convected cloaking design is proposed, based upon a simple Doppler-like correction, valid for an object at rest surrounded by a moving medium impinged by a planar wave. However, flow Mach numbers that have been used, up to 0.06, are too far from realistic aeroacoustic applications of some interest in aeronautics.

A similar approach is proposed in Iemma [7], where a correction based on a local Doppler factor is used to derive a simple correction for the metamaterial parameters, to obtain the acoustic cloaking of objects impinged by the acoustic perturbation generated by a comoving source. After the recasting of the governing equation inside the cloak in the form of the classical wave equation with additional source terms related to the metabehavior of the cloak, the problem is formulated using an original boundary integral equation. Results show a great reduction of the scattering cross section of the cloaked device for Mach number up to 0.2.

In Huang et al. [8] the problem has been addressed using a numerical optimization procedure to obtain the optimal design for the cloaking of an object immersed in a turbulent fluid. Solutions provided by this procedure are optima for the specific turbulence configuration on which the optimization is performed, requiring, for practical applications, both real time flow prediction and tunable metamaterials.

Another, and more recent, work that makes use of optimization is the one published by Ryoo and Jeon [9], who develop an acoustic analogy for the hosting medium that can take into account nonuniformity and compressibility of the background flow. In their work, the authors make use of the correction proposed by Huang et al. [6] to adapt the static acoustic cloaking design for moving fluid conditions and consider an impinging planar wave on a cylindrical object. The designed cloaking device is divided into zones and the corrective term is then optimized for each zone, giving rise to an equivalent Mach number of the part.

All these works have in common the assumption of aerodynamic impermeability of the cloak and, excluding the publication by Iemma [7], the assumption of incoming planar wave fronts.

The first attempt to overcome the major limitation of the STA, that is, the lack of formal invariance of equations under coordinate transformations in presence of flow, is presented in García-Meca et al. [10-13], where the Analogue Transformation Acoustic (ATA) approach is introduced and applied to simple test cases. Starting from the observation, first published by Visser [14], that the convective form of the wave equation has a relativistic structure, this new technique uses the existence of such an auxiliary abstract relativistic system to build an analogue space-time on which it operates and searches for relativistic analogues of laboratory phenomena. The appealing feature of the method for aeroacoustic applications lies in the possibility of handling a background aerodynamic flow (on which the acoustic perturbation travels) transforming it together with the space-time. However its potential in this field has just been sketched in the work of the group $[11,12]$ and not explored in detail. Till now, in fact, the applications of ATA in the aeroacoustic field are limited to the cloaking of a small geometric irregularity on a flat wall, the so called carpet cloaking, using a numerically obtained quasiconformal transformation as described in $[15,16]$.

After a brief review of the ATA, addressed in Section 2, and its application on the cited case in Section 3.1, the methodology is extended in Section 3.2 to consider the cloaking of a cylinder immersed in a moving hosting fluid with the incident acoustic field emitted by a point source located close to the object, thus eliminating the assumption of planar wave fronts. This is crucial for realistic aeronautical application, where the on-board noise sources cannot be considered at infinite distance from the scattering surfaces. In commenting the results, the paper tries to shed light on the physical meaning of the velocity field resulting from the application of the ATA methodology in combination with quasi-conformal transformations and its relationship with the potential flows model. The cloaking efficiency of the proposed design is analyzed in Section 4 through a campaign of numerical simulations covering a wide range of configuration for the relevant parameters.

\section{Analogue Transformation Acoustics Summary}

The Analogue Transformation Acoustics was first published in 2013 in García-Meca et al. [10] as a new framework for acoustic metamaterials design, alternative to the Standard Transformation Acoustics (STA) and successively developed in [10-13]. The interested reader is referred to those exhaustive works for a detailed description of the fundamentals of the method. Here, a brief review of the methodology and its application is given for the reader's convenience. Let us consider that the dynamic field of interest (the acoustic perturbation, in our case) is described by a virtual model defined using specific coordinates system in the laboratory space, and let this model depend on a set of physical parameters that identify its status (e.g., speed of sound, density, and local velocity). The ATA methodology relies on the identification of an analogue model using a relativistic system in the abstract space-time having the same structure of our original model in at least one coordinate system. The Lorentzian structure of the space-time system guarantees the forminvariance with respect to any space-time transformation. Once the transformation of interest is applied in the abstract space-time, the transformed system is mapped back in the laboratory space and the transformed parameters are now interpreted as the real parameters required to attain the target behavior. In the following, according to the nomenclature introduced in [10], the physical quantities pertaining to the virtual and real models are indicated with the subscripts $V$ and $R$, respectively.

In the specific application at hand, the virtual model is constituted by the equation governing the propagation of an acoustic disturbance within a moving compressible medium. Assuming the fluid to be inviscid and barotropic and the 
flow to be irrotational at $t=0$, Kelvin's theorem guarantees that the flow remains irrotational at all times. Under these assumptions, the velocity field is potential, and the mass and momentum conservation laws can be linearized in terms of small fluctuations of pressure, density, and velocity. Combining the linearized mass and momentum equations yields (details on the derivation of the equation can be found, e.g., in Visser [14])

$$
-\frac{\partial}{\partial t}\left(\frac{\rho}{c^{2}}\left(\frac{\partial \phi}{\partial t}+\mathbf{v} \cdot \nabla \phi\right)\right)+\nabla \cdot\left(\rho \nabla \phi-\frac{\rho}{c^{2}}\left(\frac{\partial \phi}{\partial t}+\mathbf{v} \cdot \nabla \phi\right) \mathbf{v}\right)=0
$$

where $\rho$ and $c$ are the local density and speed of sound associated with the background velocity field $\mathbf{v}$, whereas $\phi$ is the acoustic potential, related to the acoustic pressure by the linearized convective Bernoulli theorem $p(\mathbf{x}, t)=\rho(\partial \phi / \partial t+$ $\mathbf{v} \cdot \nabla \phi)$.

The analogue model can be obtained by rewriting the equations in terms of space-time coordinates $x^{\mu} \equiv\left(c t, x^{i}\right)$, with $0 \leq \mu \leq 3$ and $1 \leq i \leq 3$. Indicating with $\partial_{\mu}$ the covariant derivative with respect to $x^{\mu}(1)$ can be written in the compact and elegant way

$$
\partial_{\mu}\left(f^{\mu \nu} \partial_{\nu} \phi\right)=0
$$

with

$$
f^{\mu \nu}=\frac{\rho}{c^{2}}\left(\begin{array}{ccc}
-1 & \vdots & -v^{j} \\
\cdots & \cdot & \cdots \\
-v^{i} & \vdots & c^{2} \gamma^{i j}-v^{i} v^{j}
\end{array}\right) .
$$

As pointed out first by Visser in 1998 [14], the above form reveals the underlying Lorentzian structure of the acoustic space-time. Equation (2) can be written as the D'Alambertian of a scalar field in a curved Lorentzian (or pseudo-Riemannian) manifold:

$$
\frac{1}{\sqrt{-g}} \frac{\partial}{\partial \mu}\left(\sqrt{-g} g^{\mu \nu} \frac{\partial \phi}{\partial \nu}\right)=0
$$

provided that the contravariant metric tensor is given by

$$
\sqrt{-g} g^{\mu \nu}=f^{\mu \nu}
$$

being $g=\operatorname{det}\left(g_{\mu \nu}\right)$. The ATA takes advantage of the relativistic structure of (4), related to the convective equation (1) through matrix $f^{\mu \nu}$ and enables the use of an arbitrary coordinate transformation $h$, even mixing space and time, to deform the space-time in any chosen way, without the risk of loss of formal invariance

$$
h: x^{\mu} \longrightarrow \bar{x}^{\bar{\mu}}=\Lambda_{v}^{\bar{\mu}} x^{v}
$$

It is evident how the considered metric depends on the medium parameter. The procedure to obtain ATA-designed metamaterials can be summarized as follows: (i) Equation (2) is written in a physical and simple coordinate system, and a virtual (inverse) metric is defined.

$$
g^{\mu \nu}=\frac{1}{\rho_{V} c_{V}}\left(\begin{array}{ccc}
-1 & \vdots & -v_{V}^{j} \\
\cdots & \cdot & \cdots \\
-v_{V}^{i} & \vdots & c_{V}^{2} \gamma_{V}^{i j}-v_{V}^{i} v_{V}^{j}
\end{array}\right) .
$$

(ii) A desired general coordinate transformation $\bar{x}^{\bar{\mu}}=$ $h\left(x^{\mu}\right)$ is applied to (2) and the new metric $\bar{g}$ is obtained through standard tensorial transformation rule

$$
\bar{g}^{\bar{\mu} \bar{\nu}}=\Lambda_{\mu}^{\bar{\mu}} \Lambda_{\nu}^{\bar{v}} g^{\mu \nu}
$$

(iii) A second real medium with parameters $\rho_{R}, c_{R}$, and $v_{R}$ is defined from the metric $\widetilde{g}^{\tilde{\mu} \tilde{v}}$ obtained from the application of the formal invariance of (4) by imposing

$$
\sqrt{-\bar{g}} \bar{g}^{\bar{\mu} \bar{v}}=\sqrt{-\tilde{g}} \widetilde{g}^{\tilde{\mu} \tilde{v}}
$$

with

$$
\begin{gathered}
\bar{g}^{\bar{\mu} \bar{v}}=\frac{1}{\bar{\rho}_{V} \bar{c}_{V}}\left(\begin{array}{ccc}
-1 & \vdots & -\bar{v}_{V}^{j} \\
\ldots & \cdot & \ldots \\
-\bar{v}_{V}^{i} & \vdots & \bar{c}_{V}^{2} \bar{\gamma}_{V}^{i j}-\bar{v}_{V}^{i} \bar{v}_{V}^{j}
\end{array}\right), \\
\widetilde{g}^{\tilde{\mu} \tilde{\nu}}=\frac{1}{\widetilde{\rho}_{R} \widetilde{c}_{R}}\left(\begin{array}{ccc}
-1 & \vdots & -\widetilde{v}_{R}^{j} \\
\ldots & \cdot & \ldots \\
-\widetilde{v}_{R}^{i} & \vdots & \widetilde{c}_{R}^{2} \widetilde{\gamma}_{R}^{i j}-\widetilde{v}_{R}^{i} \widetilde{v}_{R}^{j}
\end{array}\right) .
\end{gathered}
$$

From (9) one can obtain the relation between parameters of the original virtual medium and those of the new real one by equating each element of the two matrices. Once realized, such a real medium will be able to reproduce the effect of the coordinate change.

Since the virtual model describing the dynamics of the convective acoustics (see (1)) cannot take into account anisotropy, the coordinate transformations that can be used 
within the ATA framework are limited to spatially conformal transformations, known to produce isotropic material parameters (provided that the starting medium is also isotropic as in the usual cases). Although, in principle, the ATA approach could be coupled to specific homogenization techniques to deal with anisotropic media (see, e.g., Torrent and Sánchez-Dehesa [17], García-Meca et al. [11], Urzhumov et al. [18], and Dupont et al. [19]), here we focus our attention on the implications related to the transformation of the convective velocity field and leave apart any further discussion about possible treatment of anisotropy. It is worth noting that the result obtained for $\mathbf{v}_{R}$ by equating term by term the components of (10) can be also obtained by applying the transformation directly to the aerodynamic velocity, $\widetilde{v}_{R}^{i}=$ $\bar{v}_{V}^{\bar{i}}=\Lambda_{i}^{\bar{i}} v_{V}^{i}($ see $[11])$.

\section{Numerical Simulations}

The methodology described in Section 2 has been applied to two test cases widely addressed in the literature: the carpet cloaking of a bumped wall and the cloaking of a circular cylinder. The virtual medium is standard air in both cases $\left(c=340 \mathrm{~m} / \mathrm{s}, \rho=1.225 \mathrm{~kg} / \mathrm{m}^{3}\right)$. A background velocity field is present in both cases, with a uniform free stream at $\mathbf{v}_{V}=120 \mathrm{~m} / \mathrm{s}$ (Mach number $M_{V}=0.35$ ). The perturbed velocity that will be used in the comparisons with the ATA-transformed field is numerically obtained using a compressible linear potential model. The first benchmark has been extensively analyzed within the framework of ATA and STA (see, e.g., $[10,11,20]$ ) and has been included to support our observations with results already assessed by several authors and well known in the research community. The second test case is a classic benchmark for STA but has never been addressed using the ATA approach. Its relevance in the present context is because the geometry of the obstacle (a typical blunt body) induces a significant perturbation of the background flow. This fact makes the aerodynamic inconsistencies derived from the straightforward application of ATA more evident than in the first experiment. To give to the reader an idea to what extent the mean flow is perturbed by the cylinder, it is worth noting that a free stream velocity of $130 \mathrm{~m} / \mathrm{s}$ (Mach number $M=0.38$, so only slightly higher than that used in the simulations) is accelerated by a cylinder of unit radius up to transonic conditions (i.e., local Mach number $\geq 1$ ), whereas the bump weakly affects the mean velocity field. ATA methodology is applied to design a cloaking device able to attain the masking of the obstacles also in presence of the background flow. All simulations are performed using the commercial finite element software COMSOL Multiphysics. The equations governing the convective propagation of the acoustic potential fluctuations $\phi$ inside $\mathscr{D}$ have been Fouriertransformed and rewritten considering a single-frequency field, thus reducing the numerical solution to a stationary problem for the Fourier coefficients of interest.

For both test cases, first the analogue transformations are numerically obtained solving a BVP as in [20] (it is essentially the classic technique used to build Laplacian-based grid for CFD simulations; see, e.g., [21]). Then, the ATA procedure is applied and the parameters of the real medium are obtained and numerically tested through dedicated aeroacoustic simulations at different frequencies. The considerations about the characteristics of the transformed background field and their consistency with the potential flow model are included after the aeroacoustic simulations.

3.1. Carpet Cloaking. In [11] an application of the method is presented, described in Section 2, to the case of the carpet cloaking of a bump on a flat wall, in the presence of a uniform mean flow parallel to the wall having velocity $v_{V}=120(\mathrm{~m} / \mathrm{s})$, that is, Mach number $M_{V}=0.35$. The shape of the bumped wall is defined in [20] (all dimensions expressed in meters):

$$
\begin{aligned}
& y=0.2 \cos ^{2}\left(\frac{\pi x}{2}\right) \quad 1 \leq x \leq 3 \\
& y=0 \quad \text { otherwise. }
\end{aligned}
$$

The metamaterial is positioned in a rectangular area $\mathscr{D}_{c}$, surrounding the bump, $1.5 \mathrm{~m}$ high and $4 \mathrm{~m}$ long. Applying the method described in [16], a quasi-conformal transformation is obtained numerically. The bumped domain is mapped in rectangular one. The former corresponds to the deformed virtual space of the ATA approach, whereas the latter is the starting physical space. The boundary value problem to be solved is

$$
\begin{aligned}
\bar{\nabla}^{2} y & =0, \quad \text { for } y \in \mathscr{D}_{c} \\
\text { with } y(\bar{a}) & =0, \\
y(\bar{b}) & =\bar{b}=b \\
\frac{\partial y}{\partial \bar{n}}(\bar{c}) & =0, \\
\frac{\partial y}{\partial \bar{n}}(\bar{d}) & =0, \\
\bar{\nabla}^{2} x & =0, \quad \text { for } x \in \mathscr{D}_{c} \\
\text { with } x(\bar{c}) & =\bar{c}=c, \\
x(\bar{d}) & =\bar{d}=d \\
\frac{\partial x}{\partial \bar{n}}(\bar{a}) & =0, \\
\frac{\partial x}{\partial \bar{n}}(\bar{b}) & =0,
\end{aligned}
$$

where $\bar{\nabla}^{2}$ denotes the differentiation in the virtual space, that is, the bumped-space, and $\bar{a}, \bar{b}, \bar{c}$, and $\bar{d}$ define the geometry of the boundary of $\mathscr{D}_{c}$ as in Figure 1.

The numerical solution of (12) and (13) yields $x=f_{1}(\bar{x}, \bar{y})$ and $y=f_{2}(\bar{x}, \bar{y})$ inside $\mathscr{D}_{c}$. The resulting distortion of the space is depicted in Figure 1. It is important to emphasize the fact that the solution is numerical, and the value of the functions $f_{1}$ and $f_{2}$ is obtained at the nodes of the computational grid and from this solution it is possible to derive the nodal values of the design parameters of the desired metamaterial. 

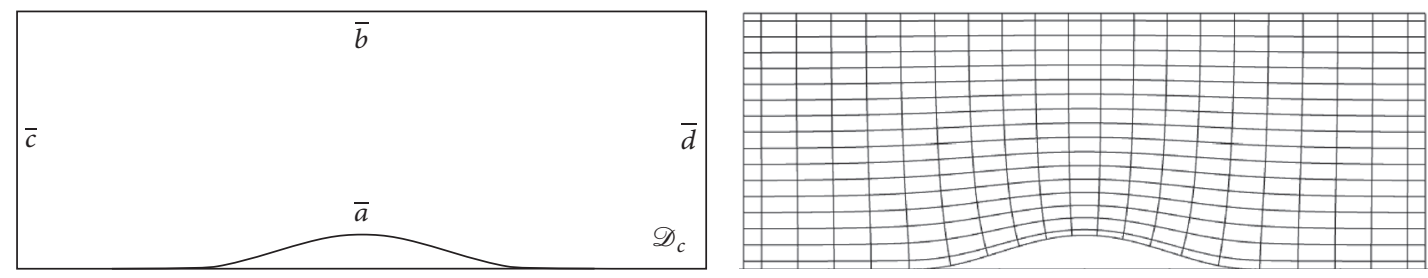

FIGURE 1: Carpet cloaking: bumped wall geometry, boundary definitions, and resulting coordinate transformation: $x=f_{1}(\bar{x}, \bar{y})$ and $y=$ $f_{2}(\bar{x}, \bar{y})$.

The distortion of the Cartesian grid shows clearly that the coordinate transformation is quasi-conformal. As demonstrated in [16], it minimizes the anisotropy required to the real metamaterial to attain the target behavior. As a consequence, anisotropy can be neglected and the real medium can be considered as isotropic, thus compatible with the virtual model adopted. The structure of the Jacobian matrix can be obtained using the properties of conformal transformation; that is, $\partial x / \partial \bar{x}=\partial y / \partial \bar{y}$ and $\partial x / \partial \bar{y}=-\partial y / \partial \bar{x}$. The inverse transformation matrix can be written as

$$
\Lambda_{\bar{\mu}}^{\mu}=\left(\begin{array}{cccc}
1 & 0 & 0 & 0 \\
0 & \frac{\partial x}{\partial \bar{x}} & \frac{\partial x}{\partial \bar{y}} & 0 \\
0 & \frac{\partial y}{\partial \bar{x}} & \frac{\partial y}{\partial \bar{y}} & 0 \\
0 & 0 & 0 & 1
\end{array}\right)=\left(\begin{array}{cccc}
1 & 0 & 0 & 0 \\
0 & \frac{\partial y}{\partial \bar{y}} & -\frac{\partial y}{\partial \bar{x}} & 0 \\
0 & \frac{\partial y}{\partial \bar{x}} & \frac{\partial y}{\partial \bar{y}} & 0 \\
0 & 0 & 0 & 1
\end{array}\right)
$$

and, hence, the transformation matrix in (6) is

$$
\Lambda_{\mu}^{\bar{\mu}}=\left(\begin{array}{cccc}
1 & 0 & 0 & 0 \\
0 & \frac{\partial x / \partial \bar{x}}{\left((\partial x / \partial \bar{x})^{2}+(\partial x / \partial \bar{y})^{2}\right)} & \frac{\partial x / \partial \bar{y}}{\left((\partial x / \partial \bar{x})^{2}+(\partial x / \partial \bar{y})^{2}\right)} & 0 \\
0 & \frac{\partial y / \partial \bar{x}}{\left((\partial x / \partial \bar{x})^{2}+(\partial x / \partial \bar{y})^{2}\right)} & \frac{\partial y / \partial \bar{y}}{\left((\partial x / \partial \bar{x})^{2}+(\partial x / \partial \bar{y})^{2}\right)} & 0 \\
0 & 0 & 0 & 1
\end{array}\right)
$$

Following the procedure described in Section 2, real medium parameters can be obtained from (9) as

$$
\begin{aligned}
c_{R} & =\frac{c_{V}}{\sqrt{(\partial x / \partial \bar{x})^{2}+(\partial x / \partial \bar{y})^{2}}}, \\
\rho_{R} & =\rho_{V}, \\
v_{R x} & =\frac{v_{V x}(\partial x / \partial \bar{x})-v_{V y}(\partial x / \partial \bar{y})}{\sqrt{(\partial x / \partial \bar{x})^{2}+(\partial x / \partial \bar{y})^{2}}}, \\
v_{R y} & =\frac{v_{V x}(\partial x / \partial \bar{y})+v_{V y}(\partial x / \partial \bar{x})}{\sqrt{(\partial x / \partial \bar{x})^{2}+(\partial x / \partial \bar{y})^{2}}} .
\end{aligned}
$$

The distribution of $c_{R} / c_{V}$ is depicted in Figure 2.

It is worth noting that the speed of sound of the soobtained real medium exhibits a behavior inversely proportional to the modulus of the transformation gradient, that is, the Jacobian of the transformation $J=\operatorname{det}(\Lambda)=$ $1 / \sqrt{(\partial x / \partial \bar{x})^{2}+(\partial x / \partial \bar{y})^{2}}$. In a static case, that is, $\vec{v}_{v}=0$, this result corresponds to what is obtained using the theoretical approach proposed by Norris [4, 5]. Indeed, according to [5], the bulk modulus of the metamaterial is $\mathscr{K}_{R}=J \mathscr{K}_{V}$, and the density is represented by the matrix $\boldsymbol{\varrho}_{R}=J\left(\boldsymbol{\Lambda} \boldsymbol{\Lambda}^{T}\right)^{-1} \rho_{0}=\mathbf{I} \rho_{0}$, confirming the equivalence of the two approaches.

Similar considerations can be made for the velocity field $\mathbf{v}_{V}$. Nevertheless, the high relevance of this aspect in aeronautical applications, where the background aerodynamics completely determines the scattering and propagation patterns, imposes additional observations. Actually, this specific point is the main driver of the present work, since it heavily influences the possibility of designing and manufacturing a metamaterial mimicking the real medium. Figure 3 shows with blue arrows the velocity field obtained by solving numerically the BVP governing the aerodynamics of a linear, incompressible potential flow. Denoting with $\Phi$ the aerodynamic potential, such that $\mathbf{v}=\nabla \Phi$, the problem is formulated as $\nabla^{2} \Phi=0$ with $\nabla \Phi \cdot \mathbf{n}=0$ on the rigid walls and $\Phi=M_{V} x^{1} / c$ at infinite distance from the obstacle, with $\mathbf{n}$ being the unit normal to the impermeable walls. Clearly, the velocity field is not uniform, due to the presence of the bump, and has its maximum intensity at the top of the bump, where the acceleration required to satisfy continuity and momentum conservation has its highest value. The same figure depicts also the velocity field $\mathbf{v}_{R}$ produced by the ATA, which has a behavior similar to that already observed for $c_{R}$, and presents its maximum modulus where the potential velocity has its minimum and vice versa. This 


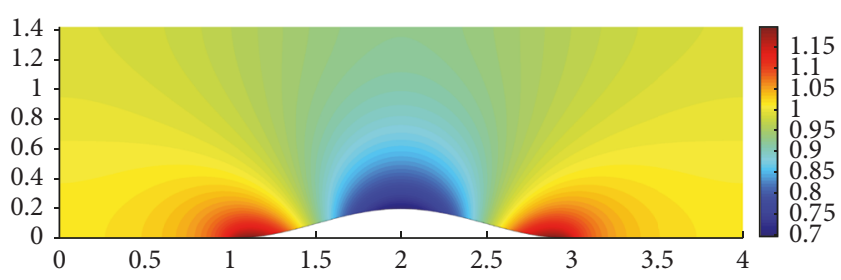

Figure 2: Field distribution of $c_{R} / c_{V}$.

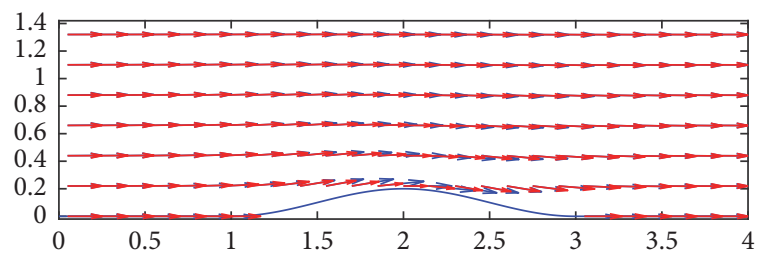

Figure 3: Comparison between the velocity field transformed with the ATA approach (red arrows) and the potential velocity field (blue arrows) around the bump inside $\mathscr{D}_{c}$.

can be explained in two steps. The lack of a perfect matching of the streamlines depends on the boundary conditions used to evaluate the two fields. Indeed, the quasi-conformal transformation has been obtained as solution of the BVP described by (12), which differs from the problem governing the potential aerodynamics only for the far-field boundary conditions. It can be easily demonstrated that the two sets of boundary conditions yield the same solution in the limit for $\mathscr{D}_{c}$ extending to infinity (the aerodynamic potential yields a uniform velocity field parallel to $x^{1}$ only for $|x| \rightarrow \infty$ ). The two solutions become identical for an infinitely wide metamaterial device, for which $x^{1}$-constant lines are distorted so as to match the streamlines of the potential flow. On the other hand, the inverse proportionality of the moduli is a consequence of the fact that the analogue transformation Jacobian matrix $\Lambda_{\mu}^{\bar{\mu}}$ is the inverse of the gradient of the solution of (12) (see (14) and (15)).

The real medium parameters obtained with the above formulation yield a metamaterial device which is able to acoustically mask the presence of the bump when impinged by an incoming acoustic perturbation. Figure 4 shows the results of the FEM simulations of the cloaking effect in presence of a background flow and for acoustic incoming fields due to a Gaussian beam impinging from the left.

The field scattered by the naked and cloaked bump are compared with the field reflected by the flat wall. When the cloak is present, the reflected wave is almost indistinguishable from the one produced by the flat wall. A tiny phase shift between the reflected wave fronts is barely detectable.

3.2. Cloaking of a Cylinder. The second test case used to assess the aeroacoustics system resulting from the application of the ATA is the cloaking of a circular cylinder impinged by the field generated by an isotropic point source. To the best of the authors' knowledge, the ATA has never been applied to such a problem, where a blunt obstacle is submerged by a moving compressible fluid. Actually, the analysis of the performance deterioration of blunt anisotropic cloaking devices in presence of a mean flow has been already addressed by several authors (see, e.g., [6-8]) who have also proposed strategies to recover, at least partially, the cloaking efficiency lost. All those contributions are based on a correction of the design obtained by STA to compensate the lack of formal invariance of the convective equations. A review of this class of approaches is outside the aim of the present work, which focuses on the aeroacoustic implications of the analogue transformation approach, and the interested reader is referred to the cited references.

The aim of the present section is the assessment of the ATA approach for obstacles inducing a significant perturbation of the aerodynamic and acoustic fields, for which most of the approximations introduced in Section 3.1 are not applicable. Indeed, in case that the geometry of the obstacle cannot be considered as a small modification of the virtual free field, the coordinate transformation obtained with the approach presented in [16] is no longer quasi-conformal. As a consequence, the ATA fails in the definition of isotropic physical parameters to define the mechanical properties of the real medium, since the anisotropy of the distorted space is no longer negligible. Figure 5 depicts this concept for the circular section. On Figure 5(a), the nomenclature of the domain occupied by the cloaking material is presented, whereas the distortion of the Cartesian grid induced by the application of (12) and (13) is shown on Figure 5(b).

The significant deviation of the grid from local orthogonality is evident, confirming that the problem statement used in Section 3.1 is not suitable to address this test case. Here, we propose a slight modification of the methodology to overtake the problem, at the cost of losing the omnidirectionality of the cloaking effect. This limitation is not to be considered an issue in the present context. Indeed, the final goal of the research is the adoption of metamaterial-based devices to change the directivity pattern of the on-board noise sources and mitigate the community noise generated by aircraft operations. In this case, the relative position of the sources (fans, turbines, compressors, and high-lift-devices) and the scattering obstacles (fuselage, wings, and empennages) is known a priori and fixed. We will examine the case presented in Figure 5, where a cylinder of radius $r$ is impinged by the noise emitted by a point source in a moving fluid with background velocity $v_{v}=120(\mathrm{~m} / \mathrm{s})\left(M_{v}=0.35\right)$ parallel to $x^{1}$ axis, The cloaking device occupies a square region $\mathscr{D}_{c}$ whose side length is $l_{1}=4 \mathrm{~m}$. It is important to emphasize the fact that the incident acoustic field is generated by an acoustic monopole close to the scattering obstacle, thus removing the assumption of planar wave fronts of the previous section and consistently with realistic aeronautical configurations. The approach proposed here is based on the identification of the spatial direction of relevance for the specific application and the solution of the Laplacian-based BVP for the orthogonal coordinate. Assuming $x^{1}$ as the direction of interest, the quasi-conformal mapping is obtained by solving the problem

$$
\bar{\nabla}^{2} y=0, \text { for } y \in \mathscr{D}_{c}
$$

with $y(\bar{a})=0$, 


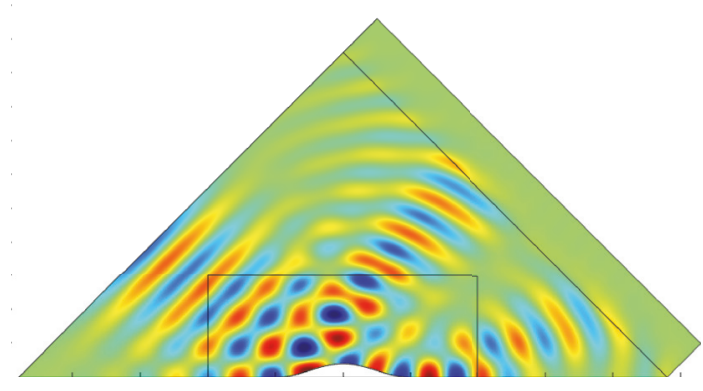

(a) Naked

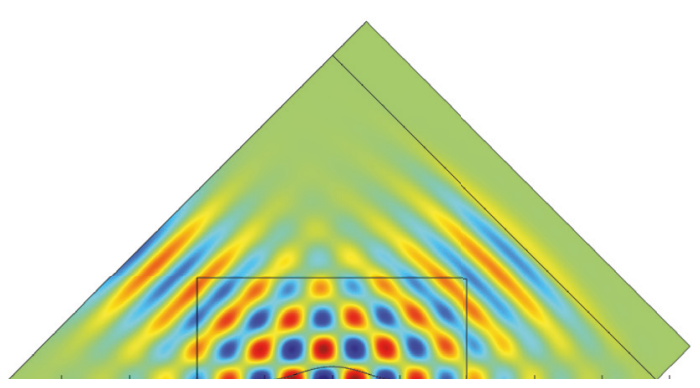

(b) Free field

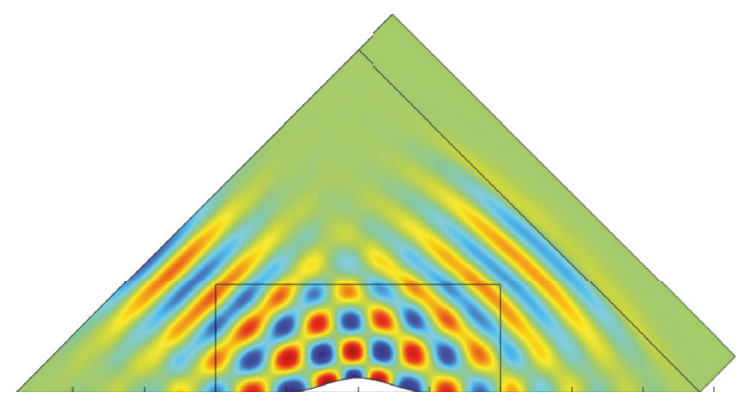

(c) Cloaked

Figure 4: Gaussian beam at $655 \mathrm{~Hz}$ impinging on a bumped wall (a), on a flat wall (b), and on a cloaked bump (c), field visualization of real part of acoustic potential $\operatorname{Re}(\phi)$.

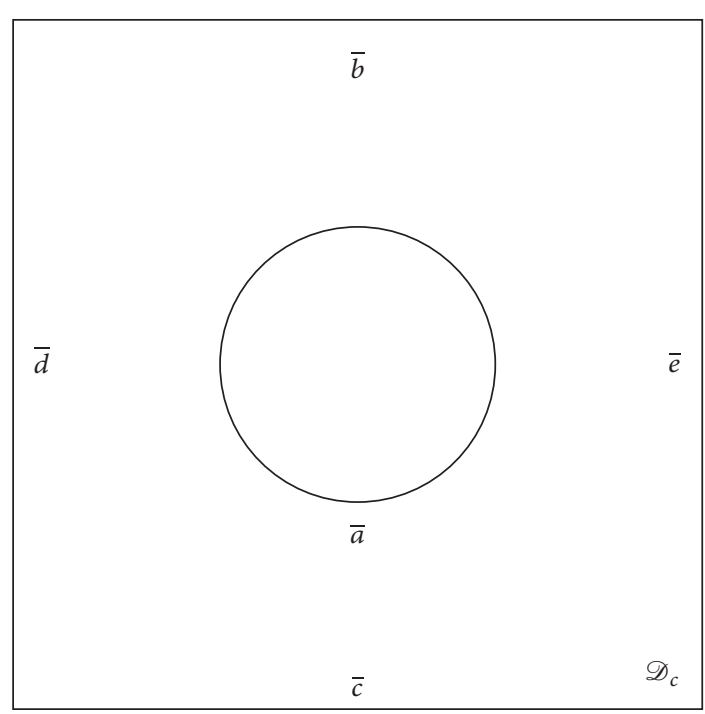

(a)

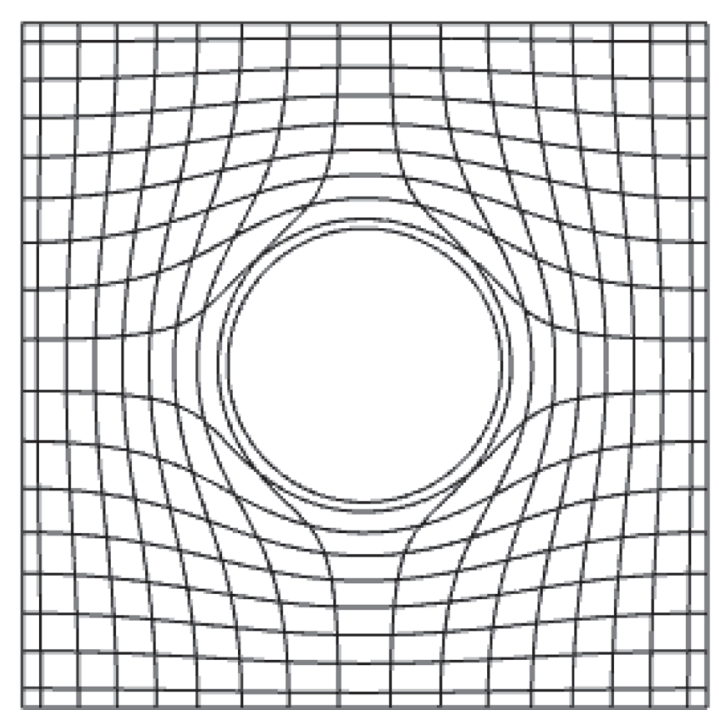

(b)

Figure 5: Nomenclature of the domain $\mathscr{D}_{c}$ occupied by the cloaking material (a) and structure of the nonconformal mapping arising from application in $\mathscr{D}_{c}$ of the method in [16].

$$
\begin{aligned}
y(\bar{b}) & =\bar{b}=b, \\
y(\bar{c}) & =\bar{c}=c \\
\frac{\partial y}{\partial \bar{n}}(\bar{d}) & =0,
\end{aligned}
$$$$
\frac{\partial y}{\partial \bar{n}}(\bar{e})=0
$$

with $\bar{\nabla}^{2}$ denoting the differentiation in the virtual space and $\bar{a}, \bar{b}, \bar{c}$, and $\bar{d}$ are defined in Figure 5. Now, we can operate as 


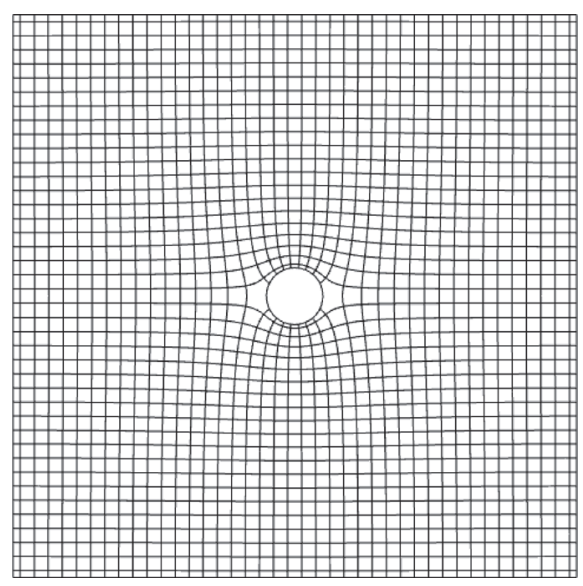

(a) Transformation

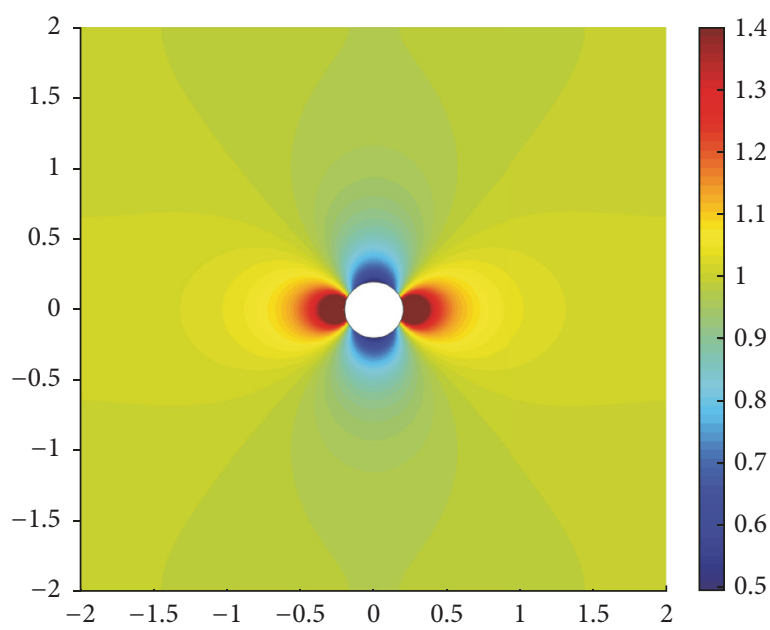

(b) $c_{R} / c_{V}$

FIGURE 6: Cloaking of a cylinder: coordinate transformation: $x=f_{1}(\bar{x}, \bar{y})$ and $y=f_{2}(\bar{x}, \bar{y})$ (a). Field distribution of $c_{R} / c_{V}$ inside $\mathscr{D}_{c}(\mathrm{~b})$.

in the previous case, exploiting the properties of conformal transformations to get

$$
\begin{aligned}
\Lambda_{\bar{\mu}}^{\mu}= & \left(\begin{array}{cccc}
1 & 0 & 0 & 0 \\
0 & \frac{\partial y}{\partial \bar{y}} & -\frac{\partial y}{\partial \bar{x}} & 0 \\
0 & \frac{\partial y}{\partial \bar{x}} & \frac{\partial y}{\partial \bar{y}} & 0 \\
0 & 0 & 0 & 1
\end{array}\right), \\
\Lambda_{\mu}^{\bar{\mu}}= & \left(\begin{array}{cccc}
1 & 0 & 0 & 0 \\
0 & \frac{\partial y / \partial \bar{y}}{\left((\partial x / \partial \bar{x})^{2}+(\partial x / \partial \bar{y})^{2}\right)} & \frac{\partial y / \partial \bar{x}}{\left((\partial x / \partial \bar{x})^{2}+(\partial x / \partial \bar{y})^{2}\right)} & 0 \\
0 & \frac{-\partial y / \partial \bar{x}}{\left((\partial x / \partial \bar{x})^{2}+(\partial x / \partial \bar{y})^{2}\right)} & \frac{\partial y / \partial \bar{y}}{\left((\partial x / \partial \bar{x})^{2}+(\partial x / \partial \bar{y})^{2}\right)} & 0 \\
0 & 0 & 0 & 1
\end{array}\right) .
\end{aligned}
$$

This transformation maps the cylinder in a flat surface aligned with the $x^{1}$ axis, thus making it invisible only to perturbations traveling along the same direction. This is the cause of the mentioned loss of omnidirectionality of the design. Figure 6 shows the coordinate mapping derived from the solution of the above problem and the distribution of $c_{R} / c_{V}$ obtained inside the cloak.

Considerations similar to those formulated in commenting Figure 4 can be made also in this case. The value of the speed of sound required to the real medium is one-half of $c_{V}$ where the local velocity has its maximum, whereas it reaches $1.5 c_{V}$ at the aerodynamic convection minima. The cloaking behavior of the metamaterial device made of the real medium is confirmed by the simulations. Figures 7 and 8 show the pressure field for the free filed and the naked and cloaked obstacle for two different relative locations of the emitting source. In Figure 7, the point source is located at $y=0$ and the cloaking effect is remarkable. The presence of the isotropic metamaterial cancels almost completely the scattering effect, with only the negligible phase shift already noticed in the first test case. From a listener point of view, such a difference would not be noted, however, with the cloaked pressure field being not identical to the incident one, and the rigorous definition of acoustic cloaking is not completely satisfied.

When the source is moved to $y \neq 0$ and the transformation is not changed accordingly, scattering cancellation deteriorates significantly. Figure 8 shows the solution obtained when the point source is moved to $y=2.5$, where the scattering effect of the obstacle is clearly visible in the whole domain surrounding the cloaking device. As already mentioned, this behavior is not surprising and consistent with the assumptions made in the application of the ATA. Another 


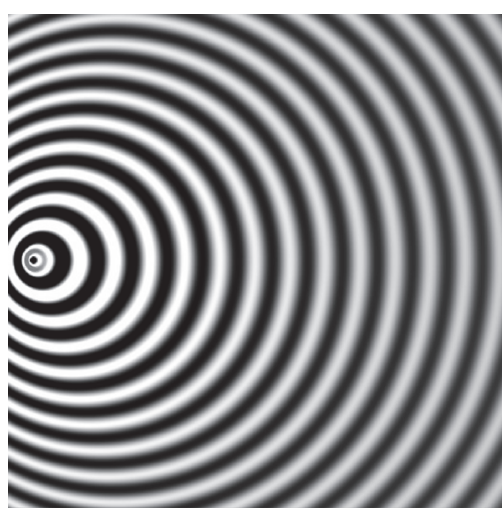

(a) Free field

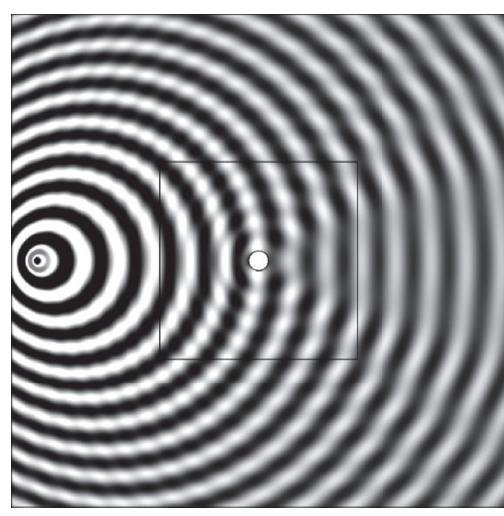

(b) Naked

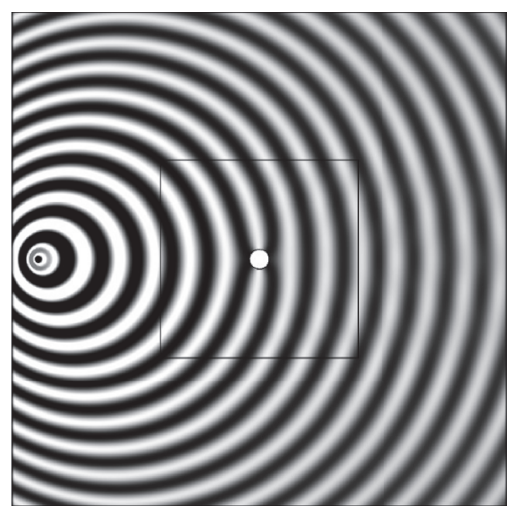

(c) Cloaked

FIGURE 7: Mass point source emitting at $655 \mathrm{~Hz}$ placed in $y=0$ impinging on a naked cylinder (a), free field (b), and cloaked cylinder (c), field visualization of real part of acoustic potential $\operatorname{Re}(\phi)$.

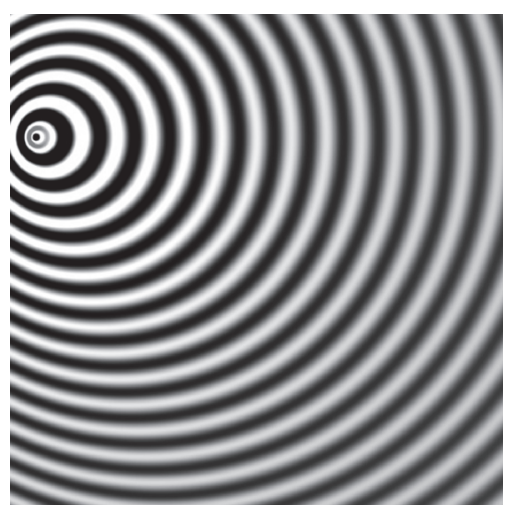

(a) Free field

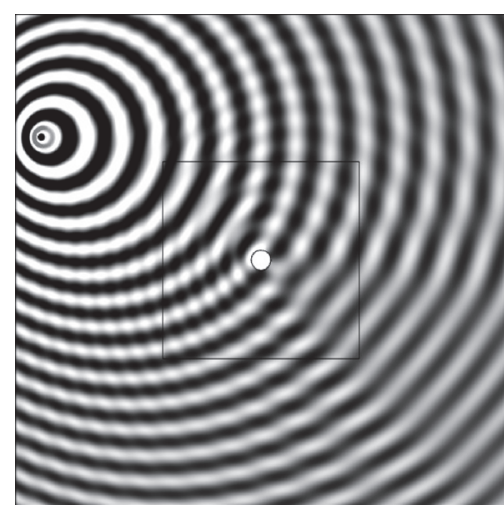

(b) Naked

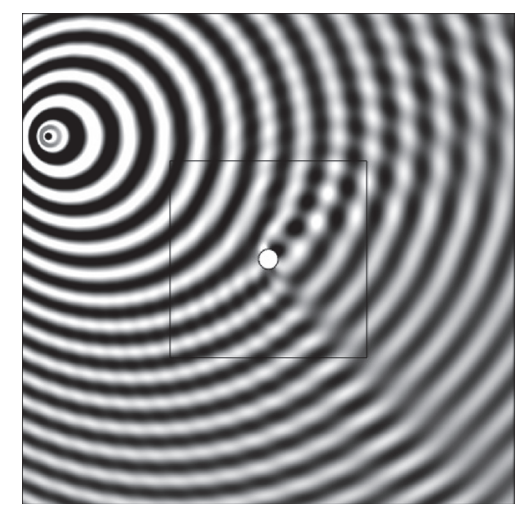

(c) Cloaked

FIGURE 8: Mass point source emitting at $655 \mathrm{~Hz}$ placed in $y=2.5$ impinging on a naked cylinder (a), free field (b), and cloaked cylinder (c), field visualization of real part of acoustic potential $\operatorname{Re}(\phi)$.

aspect that that deserves a numerical assessment is the dependence of the solution on the extension of the cloaking domain $\mathscr{D}_{c}$. The next section is dedicated to the assessment of the cloaking efficiency as a function of frequency and size of the metamaterial device.

\section{On Cloaking Efficiency}

Efficiency tests on the cloaking capability of the device are carried out for the application presented in Section 3.2, varying the frequency of the incident acoustic field and the ratio between the surface occupied by the cylindrical object and the cloak surface. The figure of merit $\sigma$ adopted to estimate the cloaking effect is the $\mathscr{L}^{1}$-norm (Lebesgue norm of order one) of the insertion loss induced by the presence of the cloaking layer, $I_{L}=20 \log _{10}\left(p_{c} / p_{f}\right)$, where subscripts $c$ and $f$ indicate the acoustic field with and without the cloaked obstacle, respectively (the general definition of the Lebesgue norm of order $p$ of a function $F$ is $\mathscr{L}^{p}=\left[\int_{\mathscr{D}}|F|^{p} d \mathscr{D}\right]^{1 / p}$; for $p=1$ it represents the mean value of the absolute value of $F$, whereas for $p=2$ it generalizes the Euclidean distance; the properties of the $\mathscr{L}^{p}$-norm for different values of $p$ are of particular interest in acoustic applications; more details can be found in Diez and Iemma [22]). This measure can be also interpreted as the $\mathscr{L}^{1}$-norm of the absolute value of the difference of sound pressure level, $L=20 \log _{10}\left(p / p_{0}\right)$, with $p_{0}=20 \mu \mathrm{Pa}$. The evaluation of the norm is extended to the portion of the computational domain occupied by the host medium, defined as $\mathscr{D}_{h}=\mathscr{D} \mid \mathscr{D}_{c}$, and is normalized using the measure of the integration domain

$$
\begin{aligned}
\sigma & =\frac{1}{\mathscr{D}_{h}} \mathscr{L}^{p}\left(I_{L}\right)=\frac{1}{\mathscr{D}_{h}} \int_{\mathscr{D}_{h}} 20 \log _{10} \frac{\left|p_{c}\right|}{\left|p_{f}\right|} d \mathscr{D} \\
& =\frac{1}{\mathscr{D}_{h}} \int_{\mathscr{D}_{h}}\left|L_{p_{c}}-L_{p_{f}}\right| d \mathscr{D} .
\end{aligned}
$$

The use of this merit factor yields results equivalent to those obtainable with any other measure based on the norm of the scattered field (see, e.g., Iemma and Burghignoli [23], Iemma [7], or Dupont et al. [19]). The measure of the computational domain occupied by the host medium is used as normalization factor.

In Figure 9, values of $\sigma$ are reported for reduced frequencies $1.099 \leq k r \leq 36.637$ with $k r=4 \pi f r / c, f=300$, 


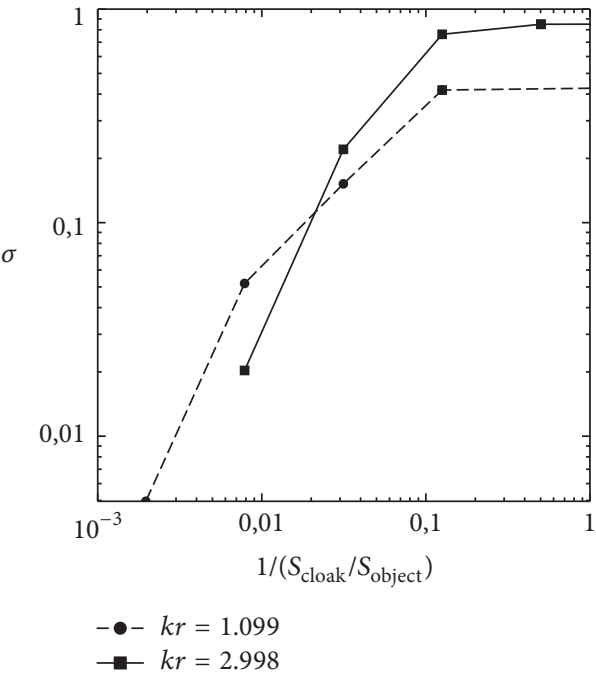

(a) $300 \mathrm{~Hz}$

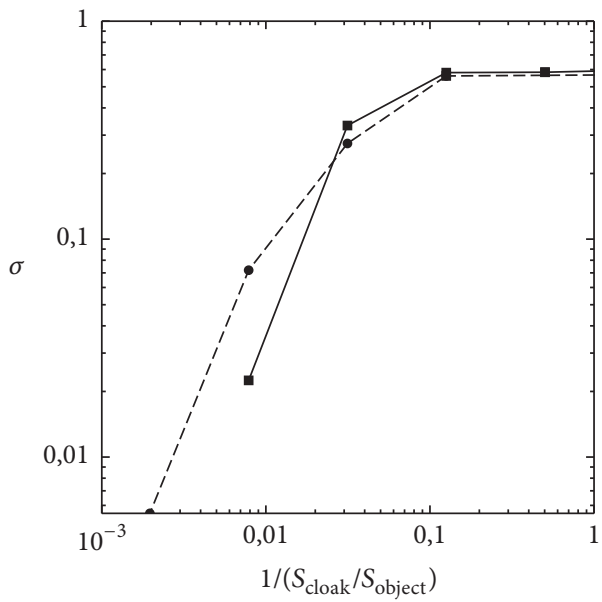

- $-k r=3.297$

$\rightarrow-k r=6.595$

(c) $900 \mathrm{~Hz}$

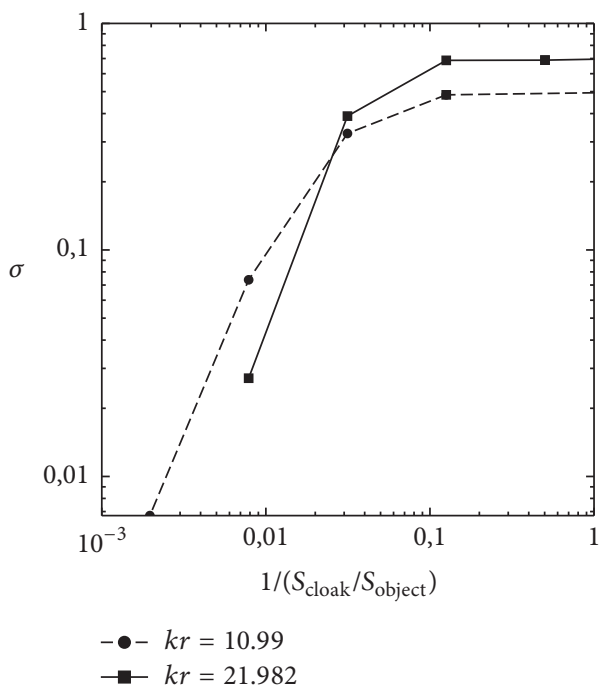

(e) $3000 \mathrm{~Hz}$

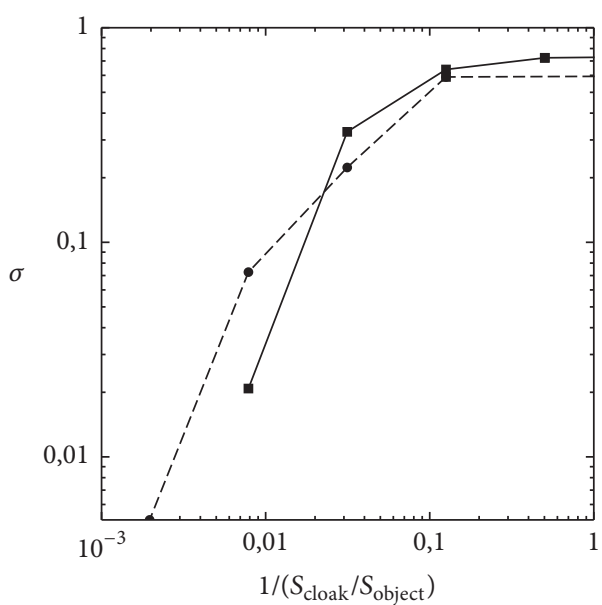

$\begin{aligned}-\bullet r & =2.399 \\ -k r & =4.799\end{aligned}$

(b) $655 \mathrm{~Hz}$

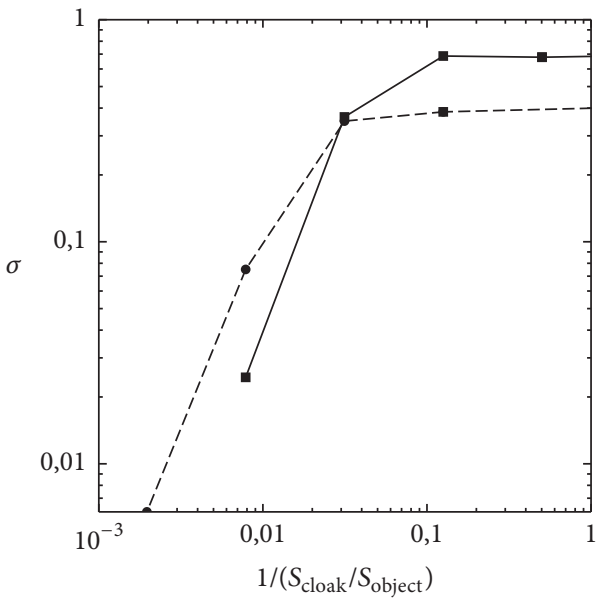

- $-k r=5.495$

- $k r=10.99$

(d) $1500 \mathrm{~Hz}$

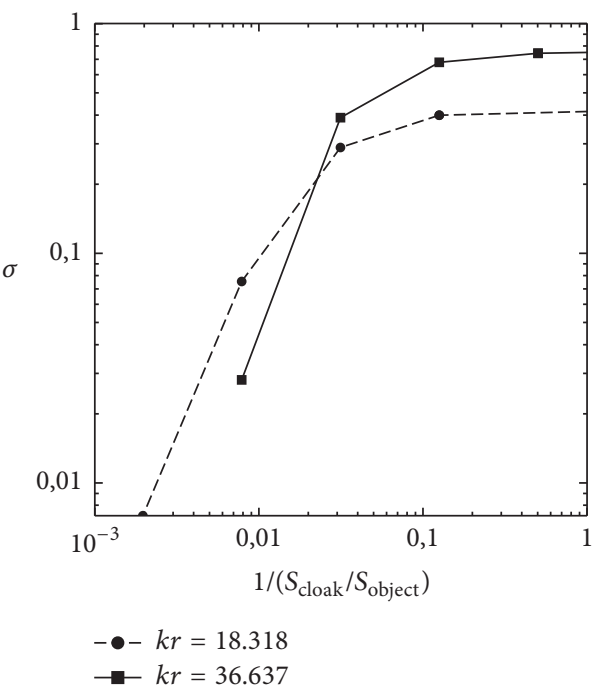

(f) $5000 \mathrm{~Hz}$

Figure 9: Values of $\sigma=1 / \mathscr{D} \int_{\mathscr{D}}\left|L_{p_{\text {cloak }}}-L_{p_{\text {free field }}}\right| d \mathcal{S}$ for six test frequencies and two sizes of cloaked cylinder at various surface ratio $S_{\text {cloak }} / S_{\text {object. }}$. 


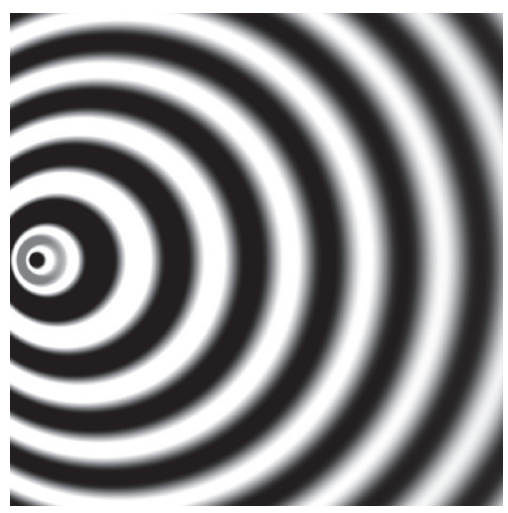

(a) Free field

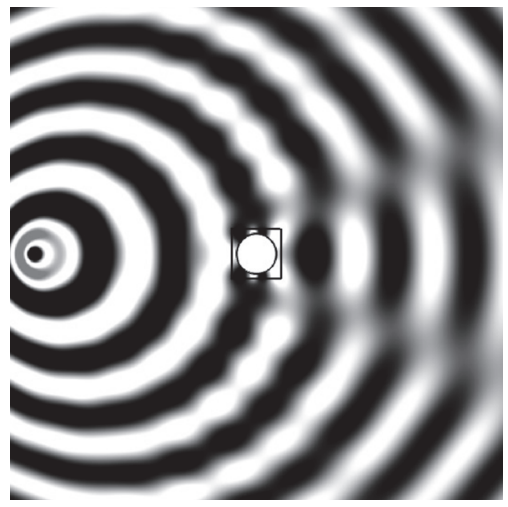

(d) $S_{\text {cloak }} / S_{\text {object }} \simeq 1, k r=2.998$

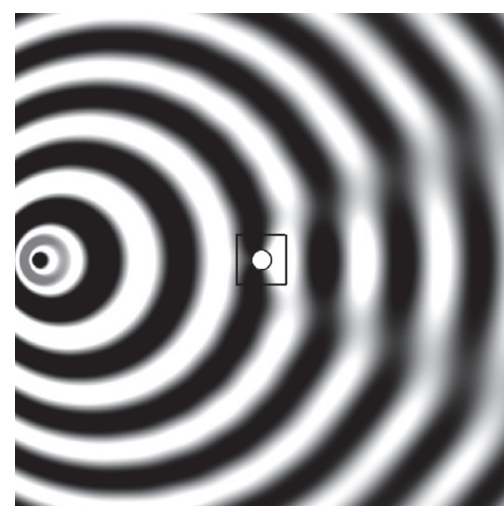

(b) $S_{\text {cloak }} / S_{\text {object }} \simeq 7, k r=1.099$

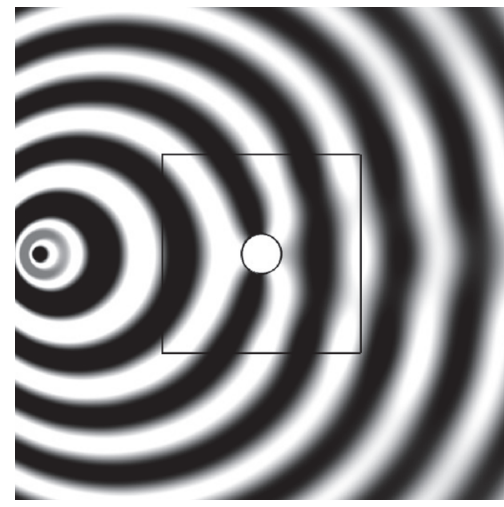

(e) $S_{\text {cloak }} / S_{\text {object }} \simeq 31, k r=2.998$

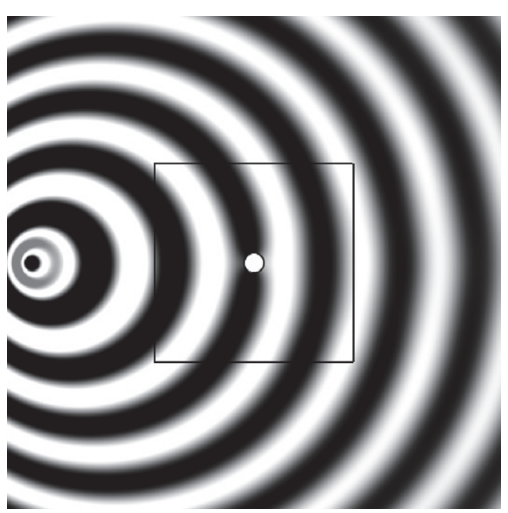

(c) $S_{\text {cloak }} / S_{\text {object }} \simeq 126, k r=1.099$

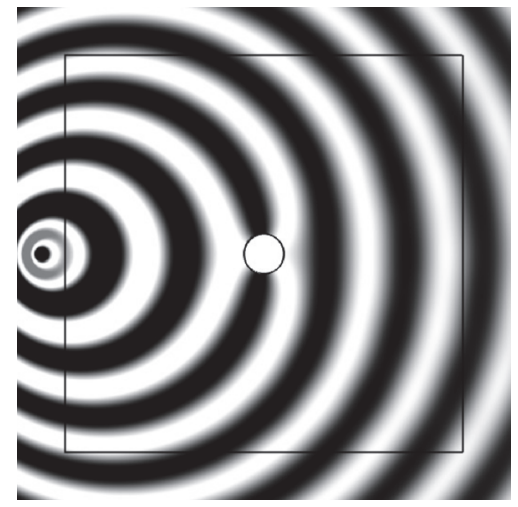

(f) $S_{\text {cloak }} / S_{\text {object }} \simeq 126, k r=2.998$

Figure 10: Acoustic potential $\phi$ field visualization at $300 \mathrm{~Hz}$ : free field (a) is compared with a small ((b) and (d)) and a big ((c), (e), and (f)) surface ratio configuration.

$655,900,1500,3000$, and $5000 \mathrm{~Hz}$, and $r=0.2$ and $0.4 \mathrm{~m}$. The value of $\sigma$ is plotted as a function of the surface ratio $S_{\text {object }} / S_{\text {cloak }}$ on a log-log scale. The analysis of results reveals that the efficacy of the device appears to be broadband also in presence of a mean aerodynamic flow, with the values of $\sigma$ being in the same range for all the analyzed frequencies. Perfect scattering cancellation is achieved asymptotically in the limit for ratio $S_{\text {object }} / S_{\text {cloak }}$ tending to zero (i.e., for a cloak covering the entire space). As anticipated, this result confirms that a metamaterial design based on ATA cannot guarantee the perfect cloaking in presence of anisotropy induced by the transformation.

The exam of the pressure fields in Figures 10-15 shows clearly this behavior in terms of nondimensional reduced frequency $k r$ for the first quart of the audible range. A high value of the surface ratio $S_{\text {cloak }} / S_{\text {object }}$ is needed in order to obtain a significant scattering abatement, with distortions of the primary field almost undetectable for the highest values of the ratio. The need for such a large cloaking surface drastically limits the applicability of this design method. This is particularly true in the aeronautical engineering context, where size, weight, and shape constraints are critical. To overcome the limitation of applicability of the method only to very small objects, a modification is needed in the way the coordinate transformation is obtained, for example, extending the ATA formulation to deal with anisotropy. This aspect currently represents the main research effort of the authors and their collaborators and will be the core activity in the future development of the method.

Finally, some consideration about the consistency of the transformed aerodynamics can be made also for the second test case. Figure 16 shows the comparison of the mean flow velocity around the circular cylinder. The characteristics already observed for the carper cloaking of the bump are here emphasized by the blunt geometry of the obstacle.

The deviation of the velocity field arising from the analogue transformation from the potential model is evident in most of the domain $\mathscr{D}_{c}$. This incompatibility of the ATA background velocity with the equations governing the aerodynamics in $\mathscr{D}_{c}$ imposes some discussion in view of the realistic exploitation of the potential of the method in aeroacoustics. First, it must be emphasized one more time that this aspect is not a limitation of the ATA, but it depends on the properties of the coordinate transformation used. In this respect, the authors are currently deeply involved in the search for specific space-time transformations able to limit or overtake completely this drawback. Having said this, it is reasonable to ask what chances a metamaterial device accomplishing the required features has to be actually manufactured. The first comment that can be done is that 


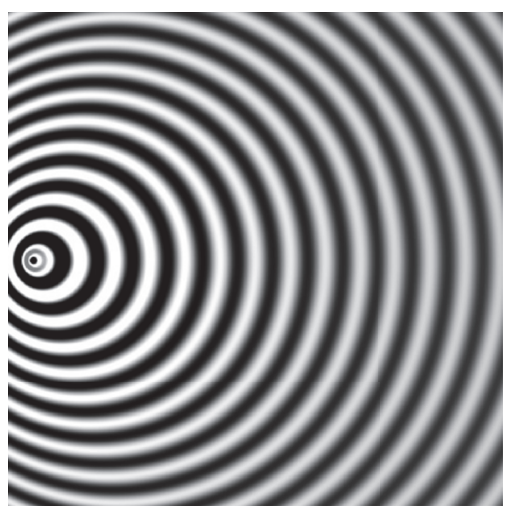

(a) Free field

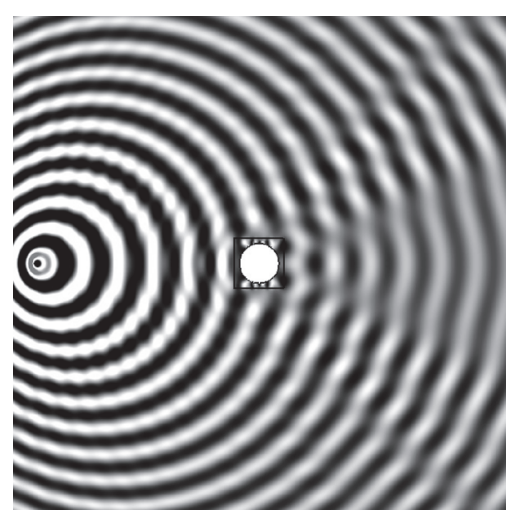

(d) $S_{\text {cloak }} / S_{\text {object }} \simeq 1, k r=4.799$

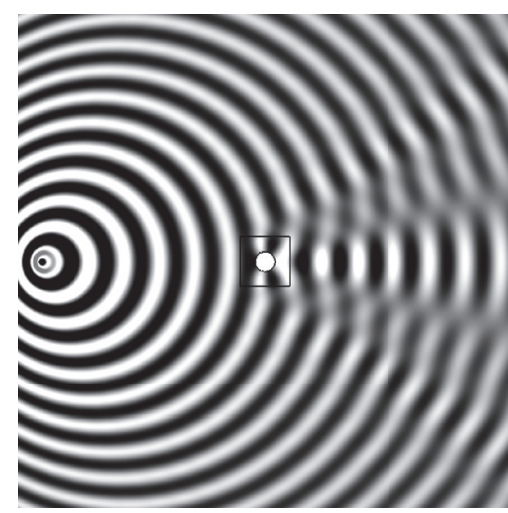

(b) $S_{\text {cloak }} / S_{\text {object }} \simeq 7, k r=2.399$

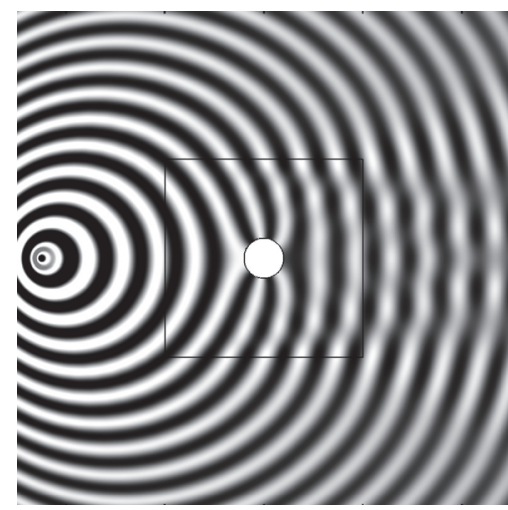

(e) $S_{\text {cloak }} / S_{\text {object }} \simeq 31, k r=4.799$

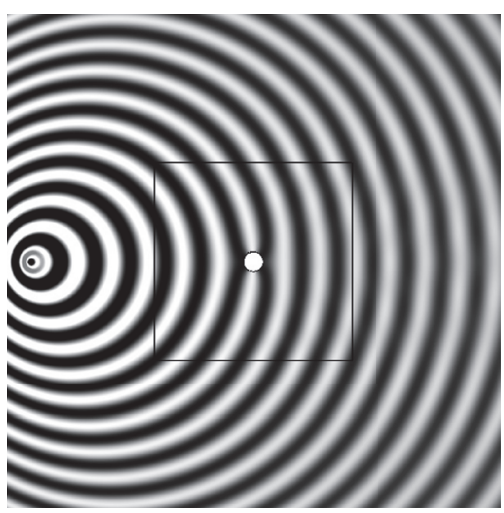

(c) $S_{\text {cloak }} / S_{\text {object }} \simeq 126, k r=2.399$

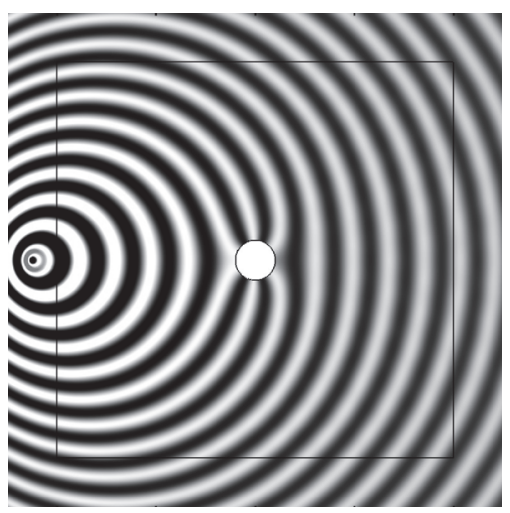

(f) $S_{\text {cloak }} / S_{\text {object }} \simeq 126, k r=4.799$

FIGURE 11: Acoustic potential $\phi$ field visualization at $655 \mathrm{~Hz}$ : free field (a) is compared with a small ((b) and (d)) and a big ((c), (e), and (f)) surface ratio configuration.

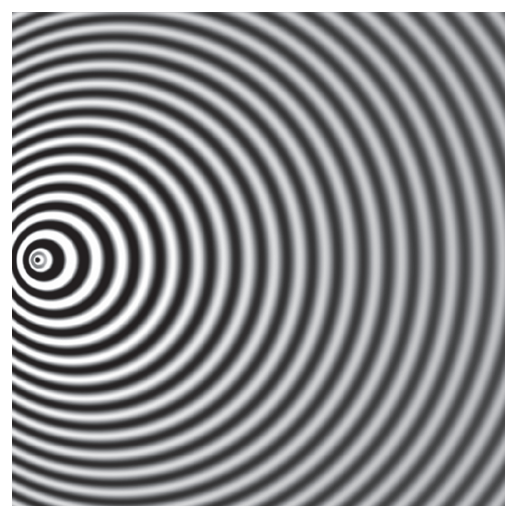

(a) Free field

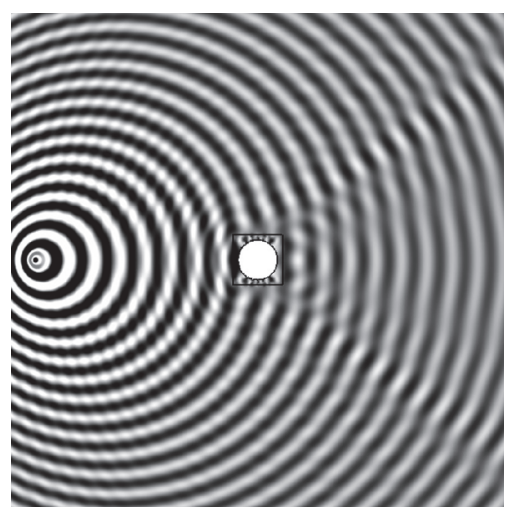

(d) $S_{\text {cloak }} / S_{\text {object }} \simeq 1, k r=6.595$

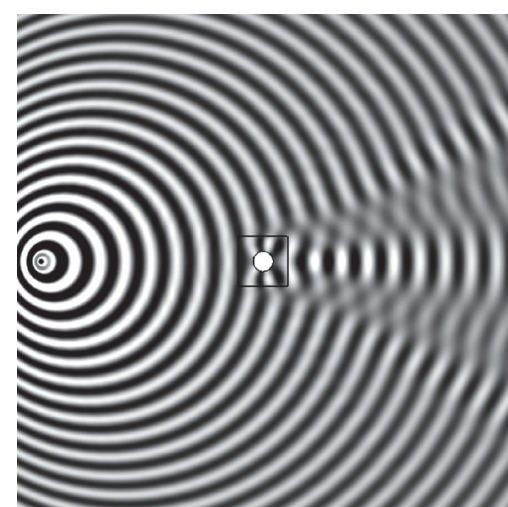

(b) $S_{\text {cloak }} / S_{\text {object }} \simeq 7, k r=3.297$

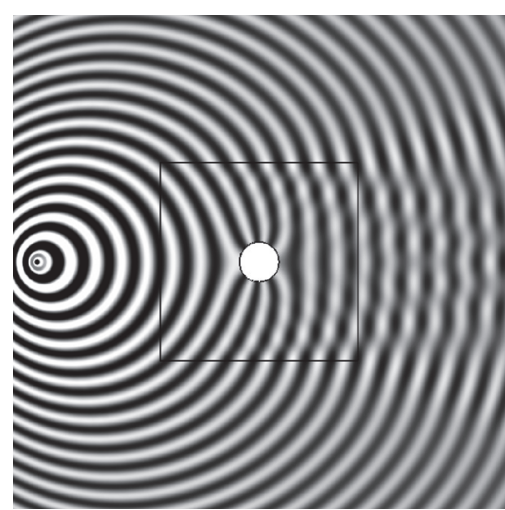

(e) $S_{\text {cloak }} / S_{\text {object }} \simeq 31, k r=6.595$

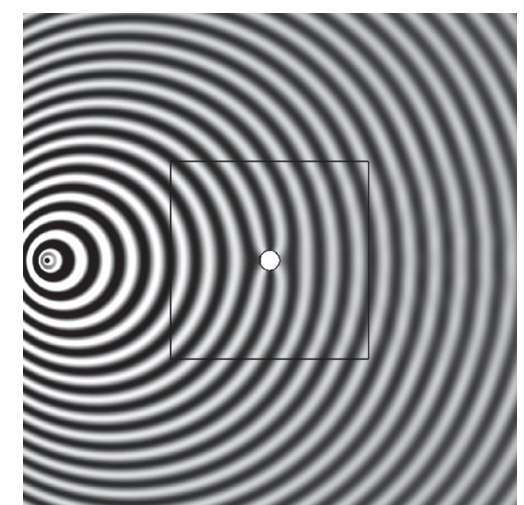

(c) $S_{\text {cloak }} / S_{\text {object }} \simeq 126, k r=3.297$

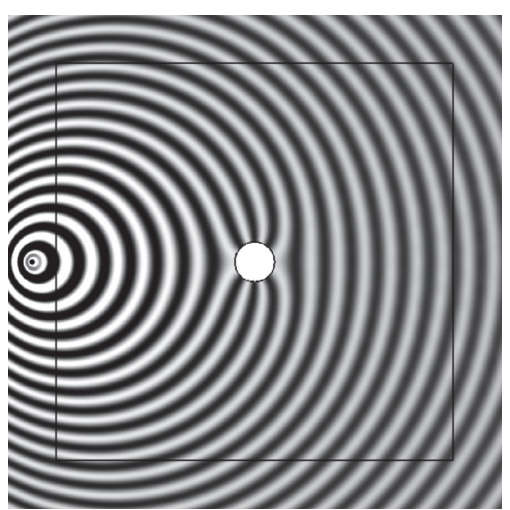

(f) $S_{\text {cloak }} / S_{\text {object }} \simeq 126, k r=6.595$

FIGURE 12: Acoustic potential $\phi$ field visualization at $900 \mathrm{~Hz}$ : free field (a) is compared with a small ((b) and (d)) and a big ((c), (e), and (f)) surface ratio configuration. 


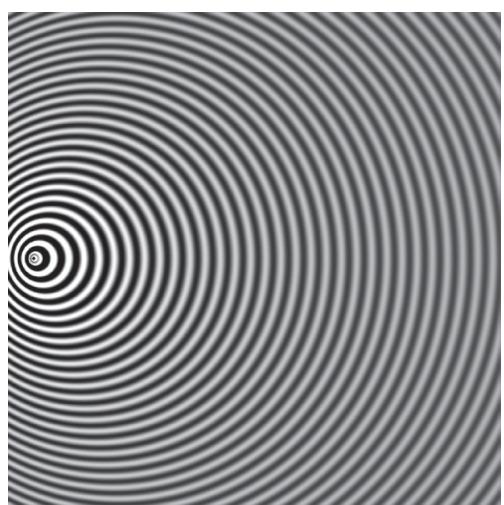

(a) Free field

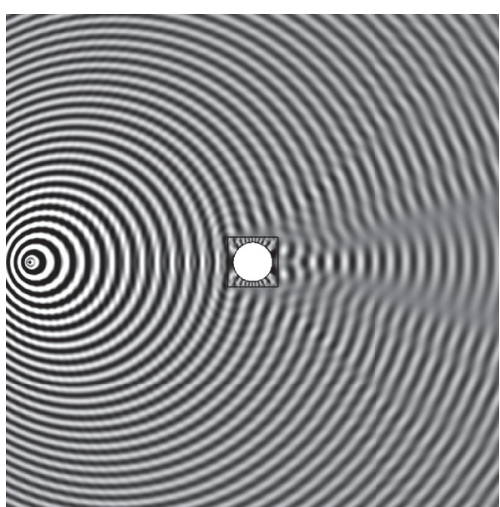

(d) $S_{\text {cloak }} / S_{\text {object }} \simeq 1, k r=10.99$

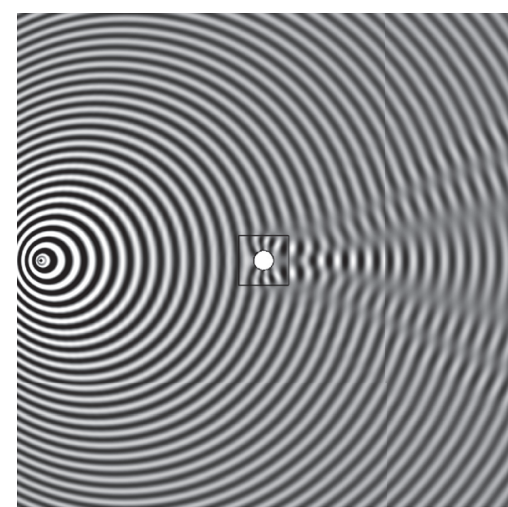

(b) $S_{\text {cloak }} / S_{\text {object }} \simeq 7, k r=5.495$

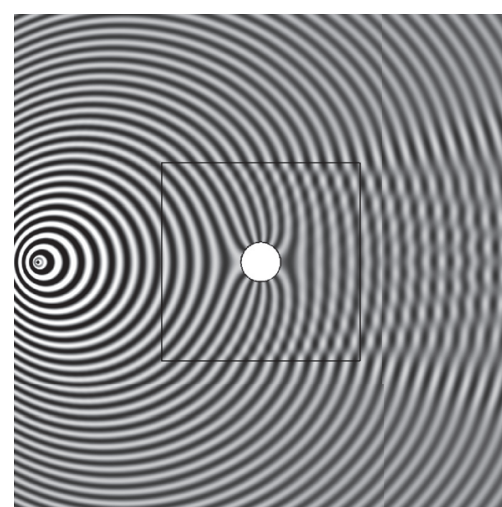

(e) $S_{\text {cloak }} / S_{\text {object }} \simeq 31, k r=10.99$

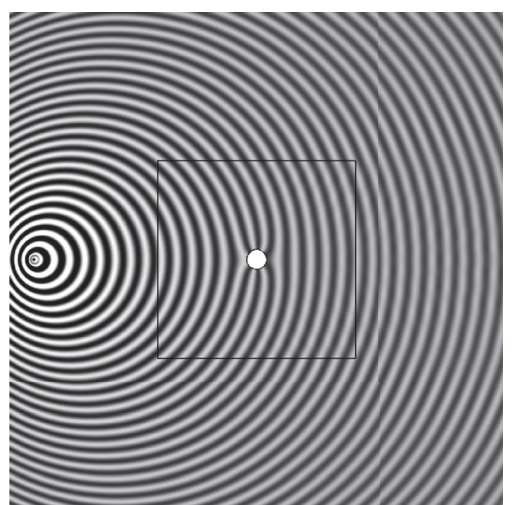

(c) $S_{\text {cloak }} / S_{\text {object }} \simeq 126, k r=5.495$

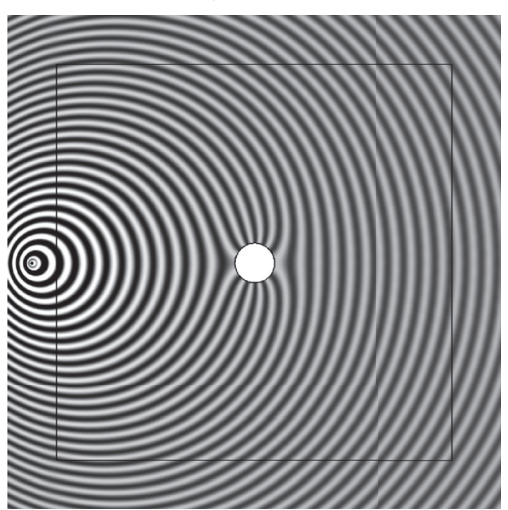

(f) $S_{\text {cloak }} / S_{\text {object }} \simeq 126, k r=10.99$

Figure 13: Acoustic potential $\phi$ field visualization at $1500 \mathrm{~Hz}$ : free field (a) is compared with a small ((b) and (d)) and a big ((c), (e), and (f)) surface ratio configuration.

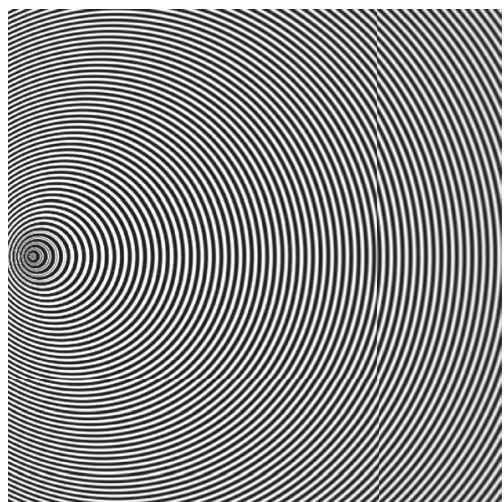

(a) Free field

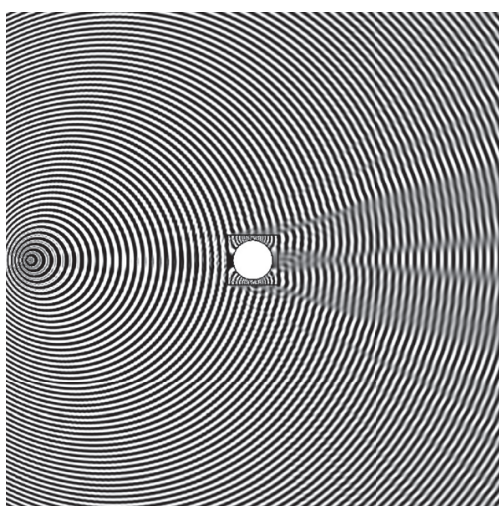

(d) $S_{\text {cloak }} / S_{\text {object }} \simeq 1, k r=21.982$

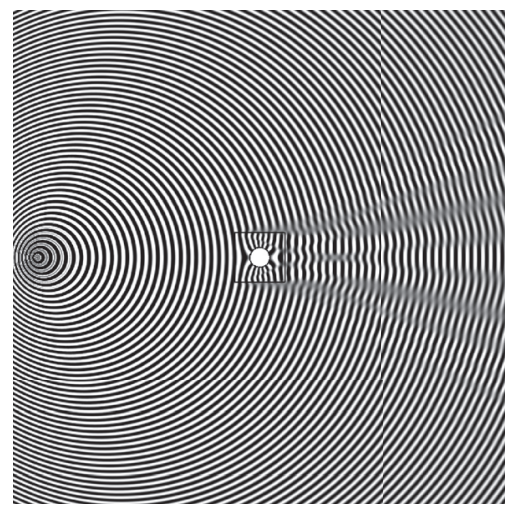

(b) $S_{\text {cloak }} / S_{\text {object }} \simeq 7, k r=10.99$

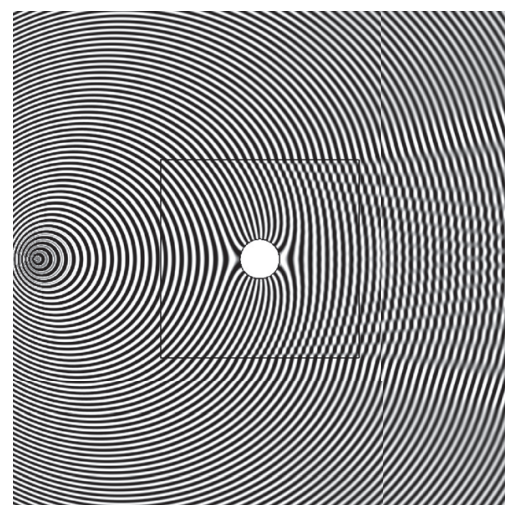

(e) $S_{\text {cloak }} / S_{\text {object }} \simeq 31, k r=21.982$

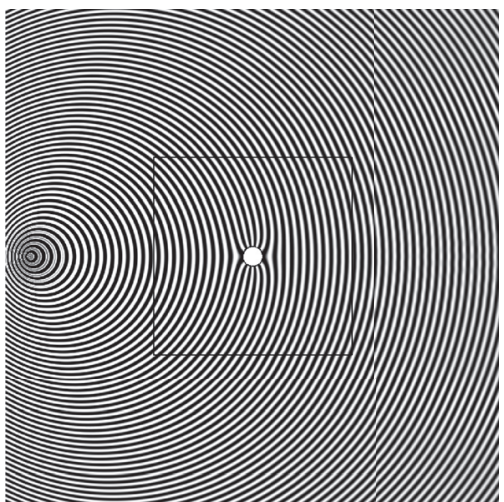

(c) $S_{\text {cloak }} / S_{\text {object }} \simeq 126, k r=10.99$

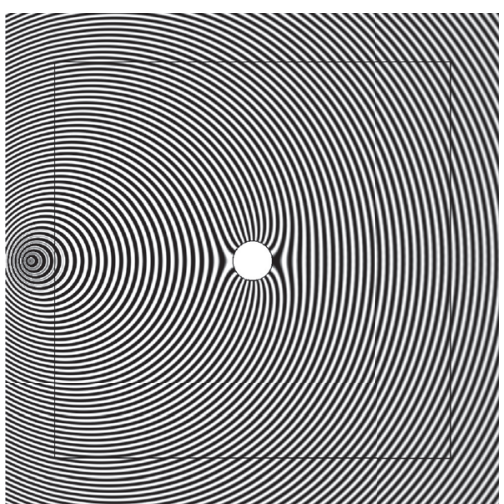

(f) $S_{\text {cloak }} / S_{\text {object }} \simeq 126, k r=21.982$

FIGURE 14: Acoustic potential $\phi$ field visualization at $3000 \mathrm{~Hz}$ : free field (a) is compared with a small ((b) and (d)) and a big ((c), (e), and (f)) surface ratio configuration. 


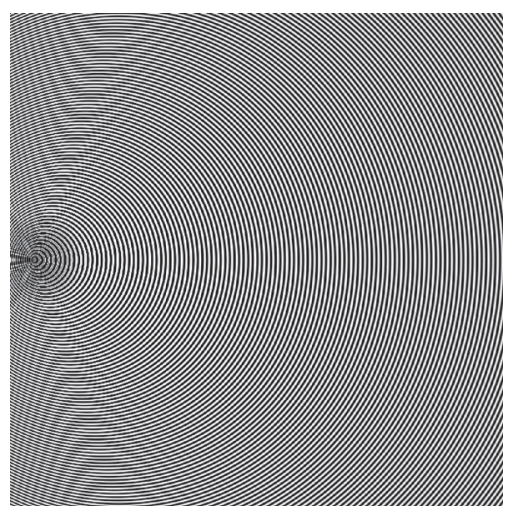

(a) Free field

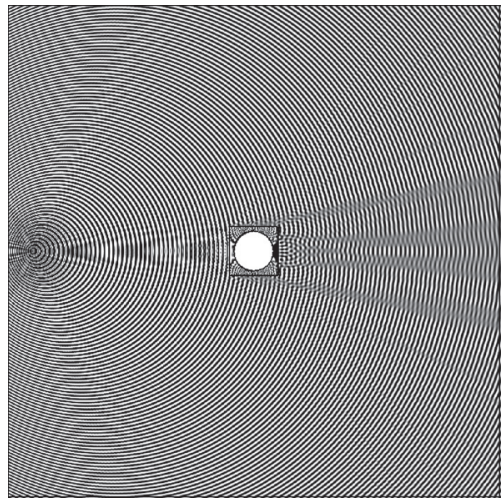

(d) $S_{\text {cloak }} / S_{\text {object }} \simeq 1, k r=36.637$

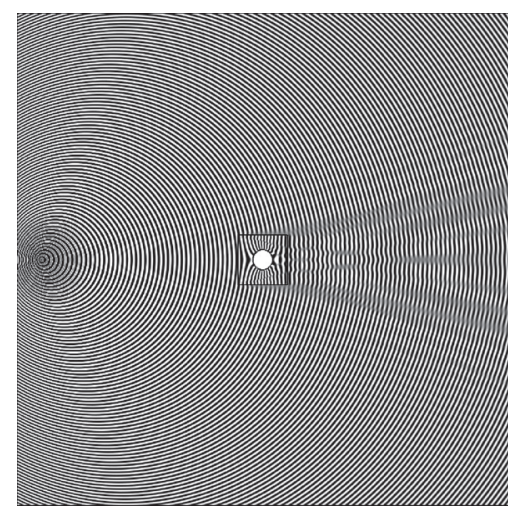

(b) $S_{\text {cloak }} / S_{\text {object }} \simeq 7, k r=18.318$

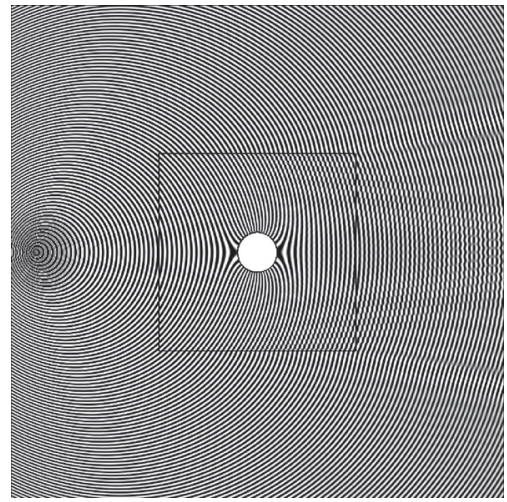

(e) $S_{\text {cloak }} / S_{\text {object }} \simeq 31, k r=36.637$

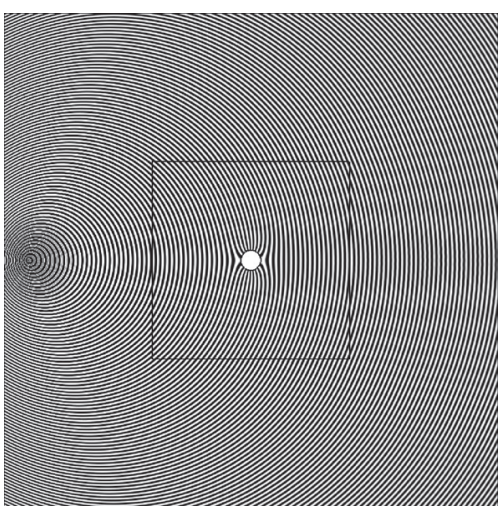

(c) $S_{\text {cloak }} / S_{\text {object }} \simeq 126, k r=18.318$

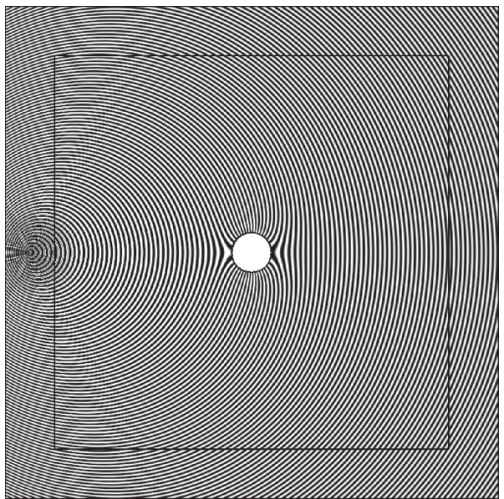

(f) $S_{\text {cloak }} / S_{\text {object }} \simeq 126, k r=36.637$

Figure 15: Acoustic potential $\phi$ field visualization at $5000 \mathrm{~Hz}$ : free field (a) is compared with a small ((b) and (d)) and a big ((c), (e), and (f)) surface ratio configuration.

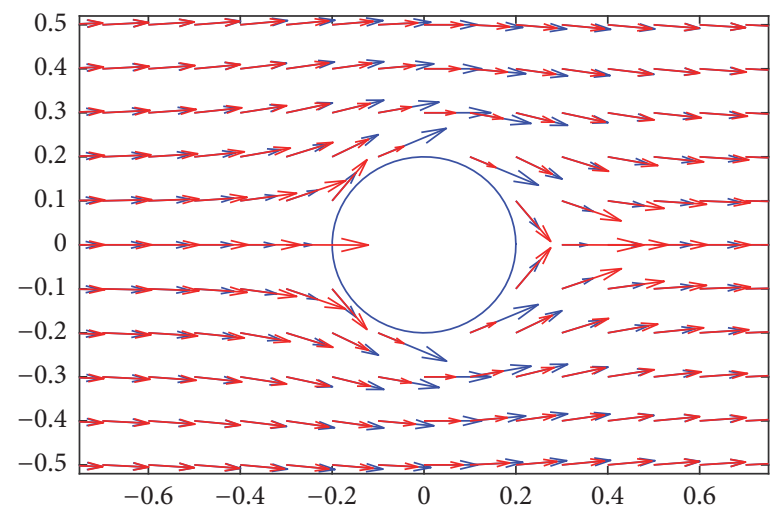

FIGURE 16: Comparison between the velocity field transformed with the ATA approach (red arrows) and the potential velocity field (blue arrows) for the circular scatterer.

a hypothetically perfectly permeable material would not be able to achieve the target behavior because of the mentioned incompatibility of the field $\mathbf{v}_{R}$ with the velocity that would appear around the obstacle, inside $\mathscr{D}_{c}$. A possible contribution to the debate about the realisability of metamaterials able to attain the desired acoustic response while negligibly affecting the aerodynamic flow can be derived from the present experience. The possibility of manufacturing a permeable metamaterial based on ATA design approach would not really help much, due to the observed and discussed properties of $\mathbf{v}_{R}$. In consideration of the results obtained, it would be better to talk about aerodynamically active metamaterials, that is, materials able to reproduce inside $\mathscr{D}_{c}$ convective terms compatible with the velocity field $\mathbf{v}_{R}$. The most straightforward way to develop a theoretical model of such active devices is, in the authors' opinion, the extension to the metamaterial concept of the acoustic analogy approach, which is based on the reformulation of the aeroacoustic equations where the standard wave operator is isolated to the left-hand side of the equation and all the convective terms are treated as acoustic sources. Several attempts in this direction have already been made and published for isotropic and anisotropic metamaterials operating with and without a flow (see, e.g., $[6-8,22,23]$ ).

\section{Conclusions}

The Analogue Transformation Acoustic method has been reviewed within the context of realistic aeroacoustic applications, where the aerodynamic convection completely determines the scattering and propagation pattern.

The focus is on the compatibility of the background flow arising inside the cloak from the application of the ATA with 
the solution of the equation governing an incompressible potential flow. The use of the potential flow model is justified by the fact that, despite its simplicity, it represents an acceptable first approximation of real flows in many aeronautical applications. In addition, for all those cases where the flow departs significantly from the potential assumption, the obtained correction can be used as initial design guess for a dedicated numerical optimization.

The ATA approach has been assessed for two test cases widely addressed in the literature. Numerical simulations of the two scenarios, that is, the carpet cloaking of a bumped wall and the cloaking of a circular cylinder, have been conducted using the finite element software to investigate on the aeroacoustic implications of the methodology.

The lack of matching with realistic, though potential, flows makes the approach of limited appeal for real world applications, at least when based on the conformal coordinate transformations proposed in the literature $[15,16]$. Furthermore, as an additional consequence of the adopted approach, the effectiveness of the metamaterial device is strongly affected by its own size making the resulting device not compatible with the stringent aerodynamic and weight constraints typical of the aeronautical applications.

Despite these limitations, the ATA still represent the only rigorous attempt to the development of techniques for the design of metamaterial operating in a flow, thus enabling the full exploitation of the metamaterial potential in aeroacoustics. Indeed, the exploitation of the Lorentzian differential geometry represents one of the most valuable contributions to the topic in the last five years. The present work represents the first step of a more comprehensive approach to combine the ATA with the substantial theoretical framework developed in aeroacoustics during the last three decades. Specifically, the use of the ATA to couple the STA with the classic aeroacoustic transformations is currently under development to extend the classic acoustic analogies and derive a general approach for the design of metamaterial devices in realistic flow conditions.

\section{Conflicts of Interest}

The authors declare that there are no conflicts of interest regarding the publication of this article.

\section{Acknowledgments}

The work was partially supported by the European Commission through Project AERIALIST (AdvancEd aircRraftnoIse-AlLeviation devIceS using meTamaterials), H2020MG-1.4-2016-2017, Project no. 723367.

\section{References}

[1] J. B. Pendry, D. Schurig, and D. R. Smith, "Controlling electromagnetic fields," American Association for the Advancement of Science. Science, vol. 312, no. 5781, pp. 1780-1782, 2006.

[2] U. Leonhardt, "Optical conformal mapping," American Association for the Advancement of Science. Science, vol. 312, no. 5781, pp. 1777-1780, 2006.
[3] S. A. Cummer and D. Schurig, "One path to acoustic cloaking," New Journal of Physics, vol. 9, no. 3, article 45, 2007.

[4] A. N. Norris, "Acoustic cloaking theory," Proceedings of the Royal Society A Mathematical, Physical and Engineering Sciences, vol. 464, no. 2097, pp. 2411-2434, 2008.

[5] A. N. Norris, "Acoustic metafluids," Journal of the Acoustical Society of America, vol. 125, no. 2, pp. 839-849, 2009.

[6] X. Huang, S. Zhong, and O. Stalnov, "Analysis of scattering from an acoustic cloak in a moving fluid," The Journal of the Acoustical Society of America, vol. 135, no. 5, pp. 2571-2580, 2014.

[7] U. Iemma, "Theoretical and numerical modeling of acoustic metamaterials for aeroacoustic applications," Aerospace, vol. 3, no. 2, article 15, pp. 1-19, 2016.

[8] X. Huang, S. Zhong, and X. Liu, "Acoustic invisibility in turbulent fluids by optimised cloaking," Journal of Fluid Mechanics, vol. 749, pp. 460-477, 2014.

[9] H. Ryoo and W. Jeon, "Effect of compressibility and nonuniformity in flow on the scattering pattern of acoustic cloak," Scientific Reports, vol. 7, no. 1, 2017.

[10] C. García-Meca, S. Carloni, C. Barceló, G. Jannes, J. SánchezDehesa, and A. Martínez, "Analogue transformations in physics and their application to acoustics," Scientific Reports, vol. 3, article 2009, 2013.

[11] C. García-Meca, S. Carloni, C. Barceló, G. Jannes, J. SánchezDehesa, and A. Martínez, "Supplementary information: analogue transformations in physics and their application to acoustics," Scientific Reports, vol. 30, 2013.

[12] C. García-Meca, S. Carloni, C. Barceló, G. Jannes, J. SánchezDehesa, and A. Martínez, "Space-time transformation acoustics," Wave Motion, vol. 51, no. 5, pp. 785-797, 2014.

[13] C. Garca-Meca, S. Carloni, C. Barceló, G. Jannes, J. SánchezDehesa, and A. Martnez, "Analogue transformation acoustics and the compression of spacetime," hotonics and Nanostructures: Fundamentals and Applications, vol. 12, no. 4, pp. 312-318, 2014.

[14] M. Visser, "Acoustic black holes: horizons, ergospheres and Hawking radiation," Classical and Quantum Gravity, vol. 15, no. 6, pp. 1767-1791, 1998.

[15] J. Hu, X. Zhou, and G. Hu, "Design method for electromagnetic cloak with arbitrary shapes based on Laplaces equation," Optical Express, vol. 17, no. 3, Article ID 001308, pp. 1308-1320, 2009.

[16] Z. Chang, X. Zhou, J. Hu, and G. Hu, "Design method for quasiisotropic transformation materials based on inverse Laplace's equation with sliding boundaries," Optics Express, vol. 18, no. 6, pp. 6089-6096, 2010.

[17] D. Torrent and J. Sánchez-Dehesa, "Broadband acoustic cloaks based on the homogenization of layered materials," Wave Motion, vol. 48, no. 6, pp. 497-504, 2011.

[18] Y. Urzhumov, F. Ghezzo, J. Hunt, and D. R. Smith, "Acoustic cloaking transformations from attainable material properties," New Journal of Physics, vol. 12, no. 7, Article ID 73014, 2010.

[19] G. Dupont, S. Guenneau, O. Kimmoun, B. Molin, and S. Enoch, "Cloaking a vertical cylinder via homogenization in the mildslope equation," Journal of Fluid Mechanics, vol. 796, 2016.

[20] J. Li and J. B. Pendry, "Hiding under the carpet: a new strategy for cloaking," Physical Review Letters, vol. 101, no. 20, Article ID 203901, pp. 1-4, 2008.

[21] J. F. Thompson, Z. U. A. Warsi, and C. W. Mastin, Numerical Grid Generation: Foundation and Applications, Elsevier NorthHolland, Inc., New York, NY, USA, 1985. 
[22] M. Diez and U. Iemma, "Multidisciplinary conceptual design optimization of aircraft using a sound-matching-based objective function," Engineering Optimization, vol. 44, no. 5, pp. 591$612,2012$.

[23] U. Iemma and L. Burghignoli, "An integral equation approach to acoustic cloaking," Journal of Sound and Vibration, vol. 331, no. 21, pp. 4629-4643, 2012. 


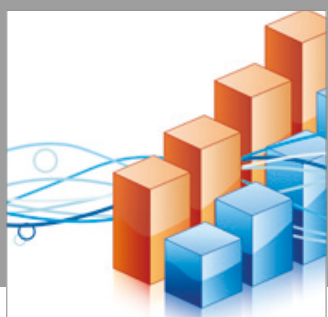

Advances in

Operations Research

vatersals

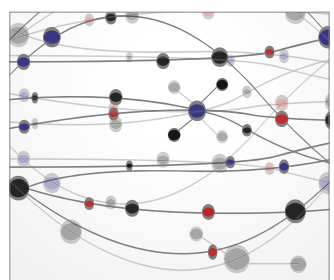

\section{The Scientific} World Journal
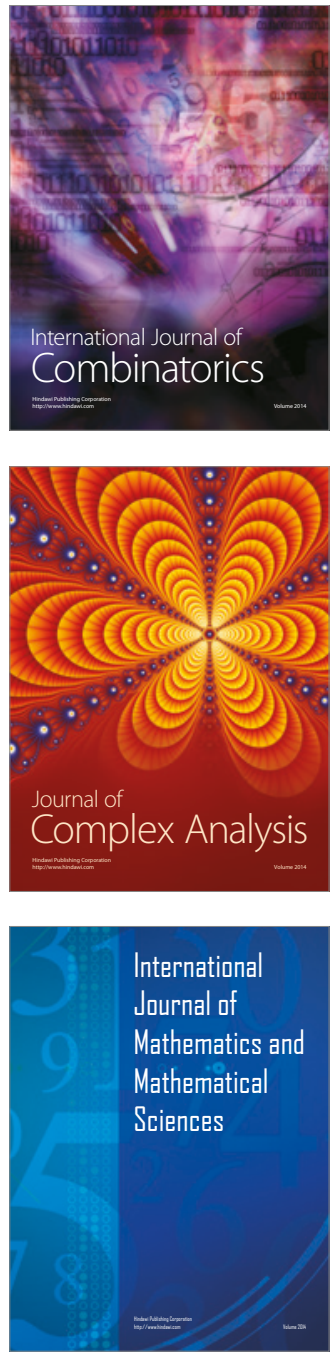
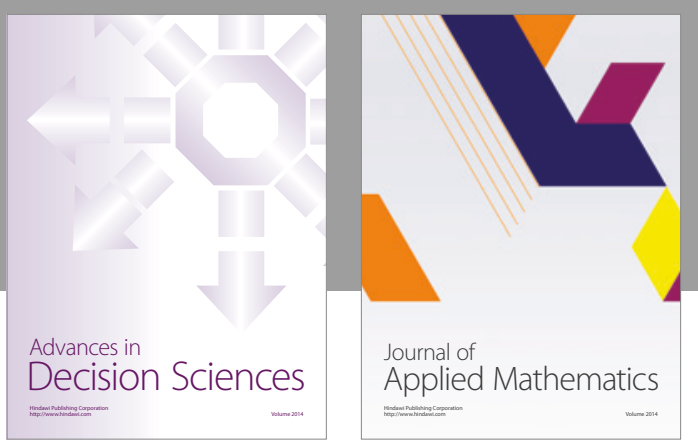

Algebra

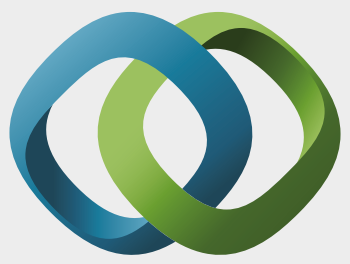

\section{Hindawi}

Submit your manuscripts at

https://www.hindawi.com
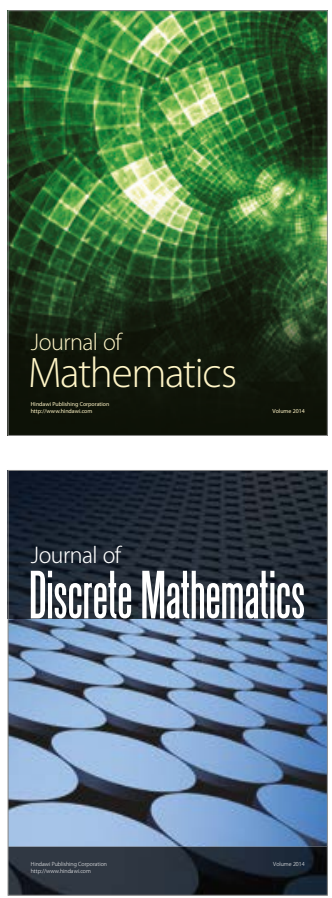

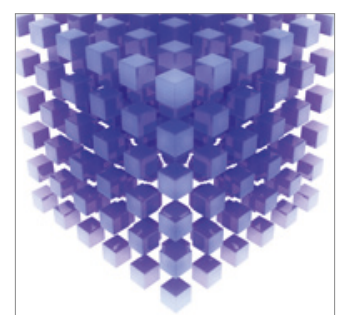

Mathematical Problems in Engineering
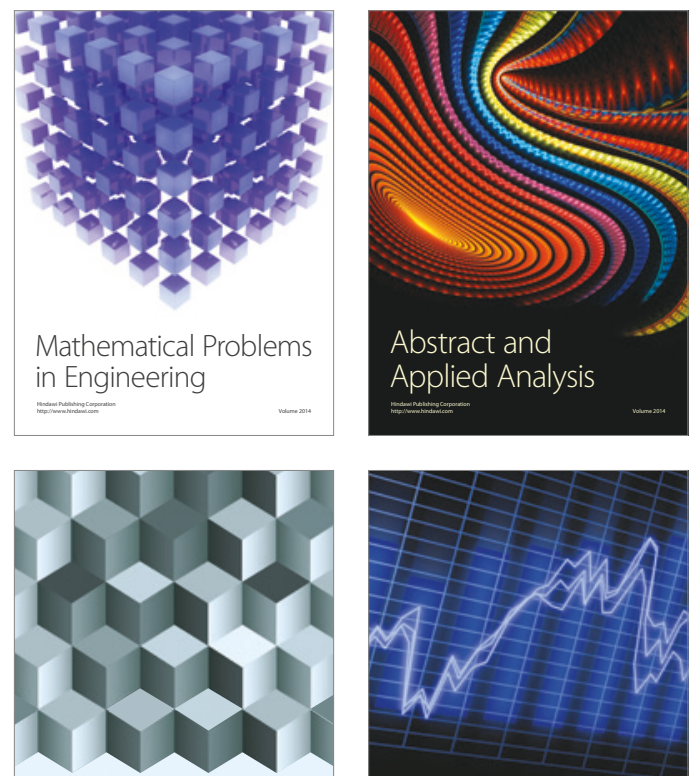

Journal of

Function Spaces

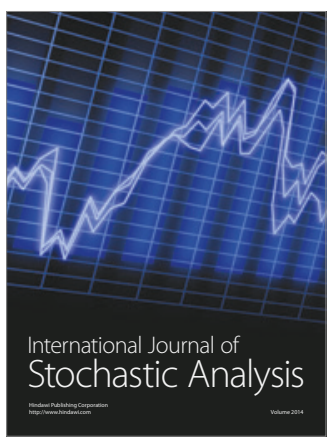

Probability and Statistics
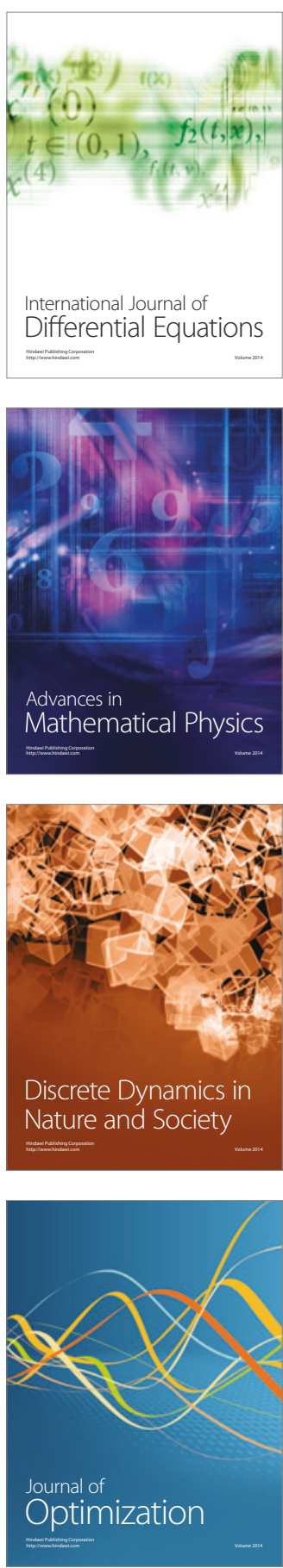